\title{
Structural Parameterizations of Clique Coloring
}

\author{
Lars Jaffke ${ }^{1}$ Paloma T. Lima ${ }^{1}$ - Geevarghese Philip ${ }^{2,3}$
}

Received: 25 September 2020 / Accepted: 25 October 2021 / Published online: 29 November 2021

(c) The Author(s) 2021

\begin{abstract}
A clique coloring of a graph is an assignment of colors to its vertices such that no maximal clique is monochromatic. We initiate the study of structural parameterizations of the CLIQUE COLORING problem which asks whether a given graph has a clique coloring with $q$ colors. For fixed $q \geq 2$, we give an $\mathscr{O}^{\star}\left(q^{\mathrm{tw}}\right)$-time algorithm when the input graph is given together with one of its tree decompositions of width tw. We complement this result with a matching lower bound under the Strong Exponential Time Hypothesis. We furthermore show that (when the number of colors is unbounded) CliQue COLORING is XP parameterized by clique-width.
\end{abstract}

Keywords Clique coloring · Parameterized algorithms · Clique-width · Treewidth

\section{Introduction}

Vertex coloring problems are central in algorithmic graph theory, and appear in many variants. One of these is CLIQUE COLORING, which given a graph $G$ and an integer $k$ asks whether $G$ has a clique coloring with $k$ colors, i.e. whether each vertex of $G$ can be assigned one of $k$ colors such that there is no monochromatic maximal clique. The notion of a clique coloring of a graph was introduced in 1991 by Duffus et al. [17],

An extended abstract of this work appeared in the proceedings of MFCS 2020 [24]. L. J. is supported by the Trond Mohn Foundation (TMS). The work was partially done while L. J. and P. T. L. were visiting Chennai Mathematical Institute.

$凶$ Paloma T. Lima

paloma.lima@uib.no

Lars Jaffke

lars.jaffke@uib.no

Geevarghese Philip

gphilip@cmi.ac.in

1 University of Bergen, Bergen, Norway

2 Chennai Mathematical Institute, Chennai, India

3 UMI ReLaX, Chennai, India 
and it behaves quite differently from the classical notion of a proper coloring, which forbids monochromatic edges. Any proper coloring is a clique coloring, but not vice versa. For instance, a complete graph on $n$ vertices only has a proper coloring with $n$ colors, while it has a clique coloring with two colors. Moreover, proper colorings are closed under taking subgraphs. On the other hand, removing vertices or edges from a graph may introduce new maximal cliques, therefore a clique coloring of a graph is not always a clique coloring of its subgraphs, not even of its induced subgraphs.

Also from a complexity-theoretic perspective, CLIQUE COLORING behaves very differently from GRAPH COLORING. Most notably, while it is easy to decide whether a graph has a proper coloring with two colors, Bacsó et al. [2] showed that it is already coNP-hard to decide if a given coloring with two colors is a clique coloring. Marx [30] later proved CLIQUE COLORING to be $\Sigma_{2}^{p}$-complete for every fixed number of (at least two) colors.

On the positive side, Cochefert and Kratsch [10] showed that the CLIQUE COLORING problem can be solved in $\mathscr{O}^{\star}\left(2^{n}\right)$ time, ${ }^{1}$ and the problem has been shown to be polynomial-time solvable on several graph classes. Mohar and Škrekovski [31] showed that all planar graphs are 3-clique colorable, and Kratochvíl and Tuza gave an algorithm that decides whether a given planar graph is 2-clique colorable [27]. For several graph classes it has been shown that all their members except odd cycles on at least five vertices (which require three colors) are 2-clique colorable [2,3,6,7,15,25,32,35]. Therefore, on these classes CLIQUE COLORING is polynomial-time solvable. Duffus et al. [17] even conjectured in 1991 that perfect graphs are 3-clique colorable, which was supported by many subclasses of perfect graphs being shown to be 2- or 3-clique colorable [1,2,9,15,17,31,32]. However, in 2016, Charbit et al. [8] showed that there are perfect graphs whose clique colorings require an unbounded number of colors.

In this work, we consider CLIQUE COLORING from the viewpoint of parameterized algorithms and complexity $[14,16]$. In particular, we consider structural parameterizations of CLIQUE COLORING by two of the most commonly used decomposition-based width measures of graphs, namely treewidth and clique-width. Informally speaking, the treewidth of a graph $G$ measures how close $G$ is to being a forest. On dense graphs, the treewidth is unbounded, and clique-width can be viewed as an extension of treewidth that remains bounded on several simply structured classes of dense graphs.

Our first main result is that $q$-CLIQUE COLORING parameterized by treewidth is fixed-parameter tractable. More precisely: we show that for any fixed $q \geq 2, q$ CLIQUE COLORING (asking for a clique coloring with $q$ colors) can be solved in time $\mathscr{O}^{\star}\left(q^{\mathrm{tw}}\right)$, where tw denotes the width of a given tree decomposition of the input graph. We also show that this running time is likely the best possible in this parameterization; we prove that under the Strong Exponential Time Hypothesis (SETH), for any $q \geq 2$, there is no $\epsilon>0$ such that $q$ - CLIQUE COLORING can be solved in time $\mathscr{O}^{\star}\left((q-\epsilon)^{\mathrm{tw}}\right)$. In fact, we rule out $\mathscr{O}^{\star}\left((q-\epsilon)^{t}\right)$-time algorithms for a much smaller class of graphs than those of treewidth $t$, namely: graphs that have both pathwidth and feedback vertex set number simultaneously bounded by $t$.

\footnotetext{
1 The $\mathscr{O}^{\star}$-notation suppresses polynomial factors in the input size, i.e. for inputs of size $n$, we have that $\mathscr{O}^{\star}(f(n))=\mathscr{O}\left(f(n) \cdot n^{\mathscr{O}(1)}\right)$.
} 
Our second main result is an XP algorithm for CLIQUE COLORING with cliquewidth as the parameter. The algorithm runs in time $n^{f(w)}$, when the input $n$-vertex graph is given with a clique-width $w$-expression and $f(w)=2^{2^{\mathscr{O}(w)}}$. The doubleexponential dependence on $w$ in the degree of the polynomial stems from the notorious property of clique colorings which we mentioned above; namely, that taking induced subgraphs does not necessarily preserve clique colorings. This results in a large amount of information that needs to be carried along as the algorithm progresses.

This algorithm raises two questions. First, if CLIQUE COLORING is FPT parameterized by clique-width even if $k$ is a priori unbounded. Second, if the triple exponential dependence on $w$ can be avoided under for instance the Exponential Time Hypothesis (ETH), also in the case when $k$ is fixed. Intuitively, a positive answer to the first question only seems feasible via a proof that all graphs of clique-width $w$ can be clique colored with at most some $g(w)$ colors, for some function $g$. However, the current literature appears to be far from providing such a result. On the other hand, hardness proofs for GRAPH COLORING parameterized by clique-width [18,19] rely on the fact that cliques require many colors while keeping the clique-width small; since cliques can be clique colored with two colors, these tricks are of no use in the setting of CLIQUE COLORING. For the second (possibly more tangible) question, one could search for an algorithm for 2- CLIQUE COLORING running in time $2^{2^{2^{o(w)}}} \cdot n^{\mathscr{O}(1)}$, or rule out the existence of such an algorithm under ETH.

The paper is organized as follows. In Sect. 2, we give an introduction to the basic concepts that are important in this work; in Sect. 3 we give the results on $q$ - CLIQUE COLORING parameterized by treewidth, and in Sect. 4 we give the algorithm for CLIQUE COLORING parameterized by clique-width.

\section{Preliminaries}

Graphs. All graphs considered here are simple and finite. For a graph $G$ we denote by $V(G)$ and $E(G)$ the vertex set and edge set of $G$, respectively. For an edge $e=u v \in$ $E(G)$, we call $u$ and $v$ the endpoints of $e$ and we write $u \in e$ and $v \in e$.

For two graphs $G$ and $H$, we say that $G$ is a subgraph of $H$, written $G \subseteq H$, if $V(G) \subseteq V(H)$ and $E(G) \subseteq E(H)$. For a set of vertices $S \subseteq V(G)$, the subgraph of $G$ induced by $S$ is $G[S]:=(S,\{u v \in E(G) \mid u, v \in S\})$; and we let $G-S:=$ $G[V(G) \backslash S]$.

For a graph $H$, we say that a graph $G$ is $H$-free if $G$ does not contain $H$ as an induced subgraph. For a set of graphs $\mathscr{H}$, we say that $G$ is $\mathscr{H}$-free if $G$ is $H$-free for all $H \in \mathscr{H}$.

For a graph $G$ and a vertex $v \in V(G)$, the set of its neighbors is $N_{G}(v):=\{u \in$ $V(G) \mid u v \in E(G)\}$. Two vertices $u, v \in V(G)$ are called false twins if $N_{G}(u)=$ $N_{G}(v)$. We say that a vertex $v$ is complete to a set $X \subseteq V(G)$ if $X \subseteq N_{G}(v)$. The degree of $v$ is $\operatorname{deg}_{G}(v):=\left|N_{G}(v)\right|$. The closed neighborhood of $v$ is $N_{G}[v]:=\{v\} \cup N_{G}(v)$. For a set $X \subseteq V(G)$, we let $N_{G}(X):=\bigcup_{v \in X} N_{G}(v) \backslash X$ and $N_{G}[X]:=X \cup N_{G}(X)$. In all these cases, we may drop $G$ as a subscript if it is clear from the context. A graph is called subcubic if all its vertices have degree at most three. 
A graph $G$ is connected if for all 2-partitions $(X, Y)$ of $V(G)$ with $X \neq \emptyset$ and $Y \neq \emptyset$, there is a pair $x \in X, y \in Y$ such that $x y \in E(G)$. A connected component of a graph is a maximal connected subgraph. A connected graph is called a cycle if all its vertices have degree two. A graph that does not contain a cycle as a subgraph is called a forest and a connected forest is a tree. In a tree $T$, the vertices of degree one are called the leaves of $T$, denoted by $\mathrm{L}(T)$, and the vertices in $V(T) \backslash \mathrm{L}(T)$ are the internal vertices of $T$. A tree of maximum degree two is a path and the leaves of a path are called its endpoints. A tree $T$ is called a caterpillar if it contains a path $P \subseteq T$ such that all vertices in $V(T) \backslash V(P)$ are adjacent to a vertex in $P$. A forest is called a linear forest if all its components are paths and a caterpillar forest if all its components are caterpillars.

A tree $T$ is called rooted, if there is a distinguished vertex $r \in V(T)$, called the root of $T$, inducing an ancestral relation on $V(T)$ : for a vertex $v \in V(T)$, if $v \neq r$, the neighbor of $v$ on the path from $v$ to $r$ is called the parent of $v$, and all other neighbors of $v$ are called its children. For a vertex $v \in V(T) \backslash\{r\}$ with parent $p$, the subtree rooted at $v$, denoted by $T_{v}$, is the subgraph of $T$ induced by all vertices that are in the same connected component of $(V(T), E(T) \backslash\{v p\})$ as $v$. We define $T_{r}:=T$.

A set of vertices $S \subseteq V(G)$ of a graph $G$ is called an independent set if $E(G[S])=$ $\emptyset$. A set of vertices $S \subseteq V(G)$ is a vertex cover in $G$ if $V(G) \backslash S$ is an independent set in $G$. A graph $G$ is called complete if $E(G)=\{u v \mid u, v \in V(G)\}$. A set of vertices $S \subseteq V(G)$ is a clique in $G[S]$ is complete. A complete graph on three vertices is called a triangle.

A graph $G$ is called bipartite if its vertex set can be partitioned into two nonempty independent sets, which we will refer to as a bipartition of $G$.

Notation for Equivalence Relations. Let $\Omega$ be a set and $\sim$ an equivalence relation over $\Omega$. For an element $x \in \Omega$ the equivalence class of $x$, denoted by $[x]$, is the set $\{y \in \Omega \mid x \sim y\}$. We denote the set of all equivalence classes of $\sim$ by $\Omega / \sim$.

Parameterized Complexity. We give the basic definitions of parameterized complexity that are relevant to this work and refer to $[14,16]$ for details. Let $\Sigma$ be an alphabet. A parameterized problem is a set $\Pi \subseteq \Sigma^{*} \times \mathbb{N}$, the second component being the parameter which usually expresses a structural measure of the input. A parameterized problem $\Pi$ is said to be fixed-parameter tractable, or in the complexity class FPT, if there is an algorithm that for any $(x, k) \in \Sigma^{*} \times \mathbb{N}$ correctly decides whether or not $(x, k) \in \Pi$, and runs in time $f(k) \cdot|x|^{c}$ for some computable function $f: \mathbb{N} \rightarrow \mathbb{N}$ and constant $c$. We say that a parameterized problem is in the complexity class XP, if there is an algorithm that for each $(x, k) \in \Sigma^{*} \times \mathbb{N}$ correctly decides whether or not $(x, k) \in \Pi$, and runs in time $f(k) \cdot|x|^{g(k)}$, for some computable functions $f$ and $g$.

The concept analogous to NP-hardness in parameterized complexity is that of W[1]hardness, whose formal definition we omit. The basic assumption is that FPT $\neq W[1]$, under which no W[1]-hard problem admits an FPT-algorithm. For more details, see $[14,16]$.

Strong Exponential TimeHypothesis. In 2001, Impagliazzo et al. [20,21] conjectured that a brute force algorithm to solve the $q$-SAT problem for every fixed $q$ which given a CNF-formula with clauses of size at most $q$, asks whether it has a satisfying 
assignment, is 'essentially optimal.' This conjecture is called the Strong Exponential Time Hypothesis, and can be formally stated as follows. (For a survey of conditional lower bounds based on SETH and related conjectures, see [36].)

Conjecture (SETH, Impagliazzo et al. [20,21]) For every $\epsilon>0$, there is a $q \in \mathbb{N}$ such that $q$ - SAT on $n$ variables cannot be solved in time $\mathscr{O}^{\star}\left((2-\epsilon)^{n}\right)$.

\subsection{Treewidth}

We now define the treewidth and pathwidth of a graph, and later the notion of a nice tree decomposition that we will use later in this work.

Definition 1 (Treewidth, pathwidth) Let $G$ be a graph. A tree decomposition of $G$ is a pair $(T, \mathscr{B})$ of a tree $T$ and an indexed family of vertex subsets $\mathscr{B}=\left\{B_{t} \subseteq\right.$ $V(G)\}_{t \in V(T)}$, called bags, satisfying the following properties.

1. $\bigcup_{t \in V(T)} B_{t}=V(G)$.

2. For each $u v \in E(G)$ there exists some $t \in V(T)$ such that $\{u, v\} \subseteq B_{t}$.

3. For each $v \in V(G)$, let $U_{v}:=\left\{t \in V(T) \mid v \in B_{t}\right\}$ be the nodes in $T$ whose bags contain $v$. Then, $T\left[U_{v}\right]$ is connected.

The width of $(T, \mathscr{B})$ is $\max _{t \in V(T)}\left|B_{t}\right|-1$, and the treewidth of a graph is the minimum width over all its tree decompositions. If $T$ is a path, then $(T, \mathscr{B})$ is called a path decomposition, and the pathwidth of a graph is the minimum width over all its path decompositions.

Let $G$ be a graph with tree decomposition $(T, \mathscr{B})$, and assume that $T$ is rooted in some node $r \in V(T)$. Then, for each node $t \in V(T)$, we let $V_{t}$ be the set of vertices of $G$ appearing in bags of the subtree of $T$ rooted at $t$, i.e. $V_{t}:=\bigcup_{s \in V\left(T_{t}\right)} B_{s}$. We let $G_{t}:=$ $G\left[V_{t}\right]$. The following notion of a nice tree decomposition allows for streamlining the description of dynamic programming algorithms over tree decompositions.

Definition 2 (Nice tree decomposition) Let $G$ be a graph and $(T, \mathscr{B})$ a tree decomposition of $G$. Then, $(T, \mathscr{B})$ is called a nice tree decomposition, if $T$ is rooted and each node is of one of the following types.

Leaf. A node $t \in V(T)$ is a leaf node, if $t$ is a leaf of $T$ and $B_{t}=\emptyset$.

Introduce. A node $t \in V(T)$ is an introduce node if it has precisely one child $s$, and there is a unique vertex $v \in V(G) \backslash B_{s}$ such that $B_{t}=B_{s} \cup\{v\}$. In this case we say that $v$ is introduced at $t$.

Forget. A node $t \in V(T)$ is a forget node, if it has precisely one child $s$, and there is a unique vertex $v \in B_{s}$ such that $B_{t}=B_{s} \backslash\{v\}$. In this case we say that $v$ is forgotten at $t$.

Join. A node $t \in V(T)$ is a join node, if it has precisely two children $s_{1}$ and $s_{2}$, and $B_{t}=B_{s_{1}}=B_{s_{2}}$. 
It is known that any tree decomposition of a graph can be transformed in linear time into a nice tree decomposition of the same width, with a relatively small number of bags.

Lemma 1 (Kloks [26]) Let $G$ be a graph on $n$ vertices, and let $k$ be a positive integer. Any width-k tree decomposition $(T, \mathscr{B})$ of $G$ can be transformed in time $\mathscr{O}(k \cdot|V(T)|)$ into a nice tree decomposition $\left(T^{\prime}, \mathscr{B}^{\prime}\right)$ of width $k$ such that $\left|V\left(T^{\prime}\right)\right|=\mathscr{O}(k \cdot n)$.

\subsection{Clique-Width, Branch Decompositions, and Module-Width}

We first define clique-width, introduced by Courcelle et al. [11], and then the equivalent measure of module-width that we will use in our algorithm. We keep the definition of clique-width slightly informal and refer to [11,12] for more details.

Let $G$ be a graph. The clique-width of $G$, denoted by $\operatorname{cw}(G)$, is the minimum number of labels $\{1, \ldots, k\}$ needed to obtain $G$ using the following four operations:

1. Create a new graph consisting of a single vertex labeled $i$.

2. Take the disjoint union of two labeled graphs $G_{1}$ and $G_{2}$.

3. Add all edges between pairs of vertices of label $i$ and label $j$.

4. Relabel every vertex labeled $i$ to label $j$.

We now turn to the definition of module-width which is based on the notion of a rooted branch decomposition.

Definition 3 (Branch decomposition) Let $G$ be a graph. A branch decomposition of $G$ is a pair $(T, \mathscr{L})$ of a subcubic tree $T$ and a bijection $\mathscr{L}: V(G) \rightarrow \mathrm{L}(T)$. If $T$ is a caterpillar, then $(T, \mathscr{L})$ is called a linear branch decomposition. If $T$ is rooted, then we call $(T, \mathscr{L})$ a rooted branch decomposition. In this case, for $t \in V(T)$, we define $V_{t}:=\left\{v \in V(G) \mid \mathscr{L}(v) \in \mathrm{L}\left(T_{t}\right)\right\}, \bar{V}_{t}:=V(G) \backslash V_{t}$, and $G_{t}:=G\left[V_{t}\right]$.

Module-width is attributed to Rao [33,34]. On a high level, the module-width of a rooted branch decomposition bounds, at each of its nodes $t$, the maximum number of subsets of $\overline{V_{t}}$ that make up the intersection of $\overline{V_{t}}$ with the neighborhood of some vertex in $V_{t}$.

Definition 4 (Module-width) Let $G$ be a graph, and $(T, \mathscr{L}$ ) be a rooted branch decomposition of $G$. For each $t \in V(T)$, let $\sim_{t}$ be the equivalence relation on $V_{t}$ defined as follows:

$$
\forall u, v \in V_{t}: u \sim_{t} v \Leftrightarrow N_{G}(u) \cap \overline{V_{t}}=N_{G}(v) \cap \overline{V_{t}}
$$

The module-width of $(T, \mathscr{L})$ is $\operatorname{mw}(T, \mathscr{L}):=\max _{t \in V(T)}\left|V_{t} / \sim_{t}\right|$. The modulewidth of $G$, denoted by $\operatorname{mw}(G)$, is the minimum module width over all rooted branch decompositions of $G$.

We introduce some notation. For a node $t \in V(T)$ and a set $S \subseteq V\left(G_{t}\right)$, we let eqc $_{t}(S)$ be the set of all equivalence classes of $\sim_{t}$ which have a nonempty intersection with $S$, and $\overline{\operatorname{eqc}}_{t}(S)$ be the remaining equivalence classes of $\sim_{t}$. Formally, eqc $c_{t}(S):=$ 
$\left\{Q \in V_{t} / \sim_{t} \mid Q \cap S \neq \emptyset\right\}$ and $\overline{\operatorname{eqc}}_{t}(S):=V_{t} / \sim_{t} \backslash$ eqc $(S)$. Moreover, for a set of equivalence classes $\mathscr{Q} \subseteq V_{t} / \sim_{t}$, we let $V(\mathscr{Q}):=\bigcup_{Q \in \mathscr{Q}} Q$.

Let $(T, \mathscr{L})$ be a rooted branch decomposition of a graph $G$ and let $t \in V(T)$ be a node with children $r$ and $s$. We now describe an operator associated with $t$ that tells us how the graph $G_{t}$ is formed from its subgraphs $G_{r}$ and $G_{s}$, and how the equivalence classes of $\sim_{t}$ are formed from the equivalence classes of $\sim_{r}$ and $\sim_{s}$. Concretely, we associate with $t$ a bipartite graph $H_{t}$ on bipartition $\left(V_{r} / \sim_{r}, V_{s} / \sim_{s}\right)$ such that:

1. $E\left(G_{t}\right)=E\left(G_{r}\right) \cup E\left(G_{s}\right) \cup F$, where $F=\left\{u v \mid u \in V_{r}, v \in V_{s},\{[u],[v]\} \in\right.$ $\left.E\left(H_{t}\right)\right\}$, and

2. there is a partition $\mathscr{P}=\left\{P_{1}, \ldots, P_{h}\right\}$ of $V\left(H_{t}\right)$ such that $V_{t} / \sim_{t}=\left\{Q_{1}, \ldots, Q_{h}\right\}$, where for $1 \leq i \leq h, Q_{i}=\bigcup_{Q \in P_{i}} Q$. For each $1 \leq i \leq h$, we call $P_{i}$ the bubble of the resulting equivalence class $\bigcup_{Q \in P_{i}} Q$ of $\sim_{t}$.

As auxiliary structures, for $p \in\{r, s\}$, we let $\eta_{p}: V_{p} / \sim_{p} \rightarrow V_{t} / \sim_{t}$ be the map such that for all $Q_{p} \in V_{p} / \sim_{p}, Q_{p} \subseteq \eta_{p}\left(Q_{p}\right)$, i.e. $\eta_{p}\left(Q_{p}\right)$ is the equivalence class of $\sim_{t}$ whose bubble contains $Q_{p}$. We call $\left(H_{t}, \eta_{r}, \eta_{s}\right)$ the operator of $t$.

Theorem 1 (Rao, Thm. 6.6 in [33]) For any graph $G, m w(G) \leq c w(G) \leq 2 \cdot m w(G)$, and given a decomposition of bounded clique-width, a decomposition of bounded module-width, and vice versa, can be constructed in time $\mathscr{O}\left(n^{2}\right)$, where $n=|V(G)|$.

\subsection{Colorings}

Let $G$ be a graph. An ordered partition $\mathscr{C}=\left(C_{1}, \ldots, C_{k}\right)$ of $V(G)$ is called a coloring of $G$ with $k$ colors, or a $k$-coloring of $G$. (Observe that for $i \in\{1, \ldots, k\}, C_{i}$ may be empty.) For $i \in\{1, \ldots, k\}$, we call $C_{i}$ the color class $i$, and say that the vertices in $C_{i}$ have color $i . \mathscr{C}$ is called proper if for all $i \in\{1, \ldots, k\}, C_{i}$ is an independent set in $G$.

A coloring $\mathscr{C}=\left(C_{1}, \ldots, C_{k}\right)$ of a graph $G$ is called a clique coloring (with $k$ colors) if there is no monochromatic maximal clique, i.e. no maximal clique $X$ in $G$ such that $X \subseteq C_{i}$ for some $i$. In this work, we study the following computational problems.

Clique Coloring

Input: $\quad$ Graph $G$, integer $k$

Question: $\quad$ Does $G$ have a clique coloring with $k$ colors?

\section{$q$ - CliQue COLORING FOR $q \geq 2$}

Input: $\quad$ Graph $G$

Question: $\quad$ Does $G$ have a clique coloring with $q$ colors?

The $q$ - COLORING and $q$ - LIST COLORING problems also make an appearance. In the former, we are given a graph $G$ and the question is whether $G$ has a proper coloring with $q$ colors. In the latter, we are additionally given a list $L(v) \subseteq\{1, \ldots, q\}$ for each vertex $v \in V(G)$, and additionally require the color of each vertex to be from its list. 
Whenever convenient, we alternatively denote a coloring of a graph with $k$ colors as a map $\phi: V(G) \rightarrow\{1, \ldots, k\}$. In this case, a restriction of $\phi$ to $S$ is the map $\left.\phi\right|_{S}: S \rightarrow\{1, \ldots, k\}$ with $\left.\phi\right|_{S}(v)=\phi(v)$ for all $v \in S$. For any $T \subseteq V(G)$ with $S \subseteq T$, we say that $\left.\phi\right|_{T}$ extends $\left.\phi\right|_{S}$.

\section{Parameterized by Treewidth}

In this section, we consider the $q$-CLIQUE COLORING problem, for fixed $q \geq 2$, parameterized by treewidth. First, in Sect. 3.1, we show that if we are given a tree decomposition of width tw of the input graph, then $q$-CLIQUE COLORING can be solved in time $\mathscr{O}^{\star}\left(q^{\mathrm{tw}}\right)$. After that, in Sect. 3.2, we show that this is tight according to SETH, by providing one reduction ruling out $\mathscr{O}^{\star}\left((2-\epsilon)^{\mathrm{tw}}\right)$-time algorithms for 2- CLIQUE COLORING and another one ruling out $\mathscr{O}^{\star}\left((q-\epsilon)^{\mathrm{tw}}\right)$-time algorithms for $q$ - CliQue COLORING when $q \geq 3$.

\subsection{Algorithm}

The algorithm is bottom-up dynamic programming along a nice tree decomposition $(T, \mathscr{B})$ of the input graph $G$. At each bag $B_{t}$, we enumerate all colorings of $G\left[B_{t}\right]$ and verify for each such coloring if it can be extended to $G_{t}$ such that there are no monochromatic maximal cliques that use a vertex from $V_{t} \backslash B_{t}$. Necessarily, we have to allow monochromatic maximal cliques $S$ that are contained inside $G\left[B_{t}\right]$, since further up in the tree decomposition, there may be a vertex $v$ that is complete to $S$. Therefore, all vertices in $S$ may receive the same color, as long as $v$ (or another such vertex) receives a different color. If on the other hand a monochromatic maximal clique has a vertex that has already been 'forgotten' at or below $t$, i.e. it is contained in $V_{t} \backslash B_{t}$, then this vertex has no neighbors in $V(G) \backslash V_{t}$; therefore, no vertex from $V(G) \backslash V_{t}$ can 'fix' this monochromatic maximal clique, and we can disregard the coloring at hand.

As a subroutine, we will have to be able to check at each bag $B_{t}$, if some subset $S \subseteq B_{t}$ contains a maximal clique in $G_{t}$. Doing this by brute force would add a multiplicative factor of roughly $2^{\text {tw }} \cdot n$ to the runtime which we cannot afford. To avoid this increase in the runtime, we use fast subset convolution ${ }^{2}$ to build an oracle $\mathbb{O}_{t}$ that, once constructed, can tell us in constant time whether or not any subset $S \subseteq B_{t}$ contains a maximal clique in $G_{t}$, for each node $t$. We give a dynamic programming algorithm that constructs such oracles for all nodes in the tree decomposition, to ensure that we can maintain a runtime that is linear in $n$. Since it suffices to construct this oracle once per node, this will infer only an additive factor of $2^{\mathrm{tw}} \cdot \mathrm{tw}^{\mathscr{O}(1)} \cdot n$ to the runtime, which does not increase the worst-case complexity for any $q \geq 2$.

Proposition 1 Let $G$ be a graph and $(T, \mathscr{B})$ a nice tree decomposition of $G$ of width tw. There is an algorithm that constructs a family of oracles $\left\{\mathbb{O}_{t}\right\}_{t \in V(T)}$ in time $2^{\text {tw }}$. $t^{\mathscr{O}(1)} \cdot|V(T)|$ that, once constructed, has the following property. For every $t \in V(T)$

\footnotetext{
${ }^{2}$ Similar ideas have been used by Cochefert and Kratsch [10] to give an $\mathscr{O}^{\star}\left(2^{n}\right)$-time algorithm for CLIQUE COLORING.
} 
and $S \subseteq B_{t}, \mathbb{O}_{t}$ answers in constant time whether or not $S$ contains a maximal clique in $G_{t}$.

Proof For each $t \in V(T)$, Let $f_{t}: 2^{B_{t}} \rightarrow\{0,1\}$ be the function defined as follows. For all $S \subseteq B_{t}$, we let

$$
f_{t}(S):= \begin{cases}1, & \text { if } S \text { contains a maximal clique in } G_{t}, \\ 0, & \text { otherwise. }\end{cases}
$$

To prove the statement, we have to show how to compute all values of $f_{t}$, for all $t \in V(T)$, within the claimed time bound.

As a first step, we show how to compute a family of functions $\left\{g_{t}: 2^{B_{t}} \rightarrow\right.$ $\{0,1\}\}_{t \in V(T)}$ such that for all $t \in V(T)$ and all $S \subseteq B_{t}, g_{t}(S)=1$ if and only if $S$ is a maximal clique in $G_{t}$. We do this by bottom-up dynamic programming, and now describe how to compute the function $g_{t}$ assuming that the functions at the children of $t$, if any, have been computed.

Leaf Node. If $t \in V(T)$ is a leaf node, then $B_{t}=\emptyset$, and there is nothing to compute.

Introduce Node. Suppose $t \in V(T)$ is an introduce node with child $s$ and let $v$ be the vertex introduced at $t$. Let $S \subseteq B_{t}$. There are two cases we have to consider, first when $v \notin S$ and second when $v \in S$. If $v \notin S$, then $S$ is a maximal clique in $G_{t}$ if and only if $S$ is a maximal clique in $G_{s}$ and $v$ is not complete to $S$. If $v \in S$, then any clique containing $S$ must be fully contained in $B_{t}$, since $v$ has no neighbors in $V_{t} \backslash B_{t}$. To summarize, we set:

$$
g_{t}(S):= \begin{cases}1, & \text { if either } v \notin S, g_{s}(S)=1, \text { and } S \nsubseteq N(v) \\ & \text { or } v \in S \text { and } S \text { is a maximal clique in } G\left[B_{t}\right] \\ 0, & \text { otherwise }\end{cases}
$$

Forget Node. If $t \in V(T)$ is a forget node with child $s$, then we have that $G_{t}=G_{s}$. Therefore, for each $S \subseteq B_{t}$, it suffices to set $g_{t}(S):=g_{s}(S)$.

Join Node. Suppose $t \in V(T)$ is a join node with children $s_{1}$ and $s_{2}$. Then, for each $S \subseteq B_{t}$, we have that $S$ is a maximal clique in $G_{t}$ if and only if it is both a maximal clique in $G_{S_{1}}$ and in $G_{S_{2}}$. Therefore, we let $g_{t}(S):=g_{s_{1}}(S) \cdot g_{s_{2}}(S)$.

Computing an entry of a function at an introduce node takes time at most tw ${ }^{\mathscr{O}(1)}$, and for a forget or join node it can be done in time $\mathscr{O}(1)$. Therefore, the family $\left\{g_{t}\right\}_{t \in V(T)}$ can be computed in time $2^{\text {tw }} \cdot t^{\mathscr{O}(1)} \cdot|V(T)|$.

For the remainder of the proof, recall that for a set $\Omega$, and two functions $\alpha$ and $\beta$ defined on $2^{\Omega}$, their subset convolution $\circledast$ is defined as: for all $S \in 2^{\Omega},(\alpha \circledast \beta)(S)=$ $\sum_{T \subseteq S} \alpha(T) \beta(S \backslash T)$. Fix some $t \in V(T)$. We define a constant function $c_{t}: 2^{B_{t}} \rightarrow$ $\{1\}$, meaning that $c_{t}(S)=1$ for all $S \subseteq B_{t}$, and construct a function $h_{t}:=g_{t} \circledast c_{t}$. Using the algorithm of Björklund et al. [4], all values of $h_{t}$ can be computed in time $2^{\mathrm{tw}} \cdot \mathrm{tw}^{\mathscr{O}(1)}$. By construction, each set $S \subseteq B_{t}$ contains $h_{t}(S)$ maximal cliques in $G_{t}$. 
We therefore obtain $f_{t}$ as:

$$
\forall S \subseteq B_{t}: f_{t}(S):= \begin{cases}1, & \text { if } h_{t}(S) \geq 1 \\ 0, & \text { otherwise }\end{cases}
$$

Computing the family of functions $\left\{f_{t}\right\}_{t \in V(T)}$ and therefore the family of oracles $\left\{\mathbb{O}_{t}\right\}_{t \in V(T)}$ this way can be done within an additional runtime of $2^{\mathrm{tw}} \cdot \mathrm{tw}^{\mathscr{O}(1)} \cdot|V(T)|$.

We are now ready to give the algorithm. We assume that we are given a width-tw tree decomposition of the input graph whose tree has $\mathscr{O}(\mathrm{tw} \cdot n)$ nodes. This requirement on the number of nodes is standard, see e.g. [14].

Theorem 2 For any fixed $q \geq 2$, there is an algorithm that given an $n$-vertex graph $G$ and a tree decomposition of $G$ of width tw which has $\mathscr{O}(t w \cdot n)$ nodes, decides whether $G$ has a clique coloring with $q$ colors in time $\mathscr{O}\left(q^{t w} \cdot t w^{\mathscr{O}(1)} \cdot n\right)$, and constructs one such coloring, if it exists.

Proof First, we transform the given tree decomposition of $G$ into a nice tree deocmposition $(T, \mathscr{B})$. This can be done in $\mathscr{O}\left(\mathrm{tw}^{2} \cdot n\right)$ time by Lemma 1 . We may assume that the bags at leaf nodes are empty, and that $T$ is rooted in some node $\mathfrak{r} \in V(T)$, and $B_{\mathfrak{r}}=\varnothing$.

We do standard bottom-up dynamic programming along $T$. Let $t \in V(T)$. A partial solution is a $q$-coloring of $G_{t}$ that satisfies one additional property. Suppose that in some coloring of $G_{t}$, there is a monochromatic maximal clique $X$ in $G_{t}$ that has some vertex $v \in V_{t} \backslash B_{t}$. Then, $v$ has no neighbors in $V(G) \backslash V_{t}$, therefore $X$ is also a maximal clique in $G$. This means that the present coloring cannot be extended to a coloring in which $X$ becomes non-maximal, and therefore we can disregard it.

In light of this, we define the table entries as follows. For each $t \in V(T)$ and function $\gamma_{t}: B_{t} \rightarrow\{1, \ldots, q\}$, we let $\operatorname{tab}\left[t, \gamma_{t}\right]=1$ if and only if there is a $q$-coloring $\gamma$ of $G_{t}$ such that

$-\left.\gamma\right|_{B_{t}}=\gamma_{t}$, and

- for each maximal clique $X$ in $G_{t}$ that is monochromatic under $\gamma, X \subseteq B_{t}$.

Since $B_{\mathfrak{r}}=\emptyset$, we can immediately observe that the solution to the instance can be read off the table entries at the root node, once computed. Throughout the following we denote by $\gamma_{\emptyset}$ the $q$-coloring defined on an empty domain.

Observation $1 G$ has a clique coloring with $q$ colors if and only if $\operatorname{tab}\left[\mathfrak{r}, \gamma_{\varnothing}\right]=1$.

As a preprocessing step, we compute the family of oracles $\left\{\mathbb{O}_{t}\right\}_{t \in V(T)}$ from Proposition 1 which will be used at forget nodes. We now show how to compute the table entries for the different types of nodes, assuming that the table entries at the children, if any, have previously been computed.

Leaf Node. If $t$ is a leaf node, then $B_{t}=\emptyset$ and we only have to consider the empty coloring. We set $\operatorname{tab}\left[t, \gamma_{\emptyset}\right]=1$. 
Introduce Node. Let $t \in V(T)$ be an introduce node with child $s$, and let $v$ be the vertex introduced at $t$, i.e. we have that $B_{t}=B_{s} \cup\{v\}$. Since $V_{t} \backslash B_{t}=\left(V_{t} \backslash\{v\}\right) \backslash\left(B_{t} \backslash\{v\}\right)=$ $V_{s} \backslash B_{s}$, and since $v$ has no neighbors in $V_{t} \backslash B_{t}$ by the properties of a tree decomposition, it is clear that a coloring of $G_{t}$ has a monochromatic maximal clique with a vertex in $V_{t} \backslash B_{t}$ if and only if its restriction to $V_{s}$ is a coloring of $G_{s}$ that has a monochromatic maximal clique with a vertex in $V_{s} \backslash B_{s}$. Therefore, for each $\gamma_{t}: B_{t} \rightarrow\{1, \ldots, q\}$, we simply let $\operatorname{tab}\left[t, \gamma_{t}\right]=1$ if and only if $\operatorname{tab}\left[s,\left.\gamma_{t}\right|_{B_{s}}\right]=1$.

Join Node. Let $t \in V(T)$ be a join node with children $s_{1}$ and $s_{2}$ and recall that $B_{t}=B_{s_{1}}=B_{s_{2}}$. In this case, for any $\gamma_{t}: B_{t} \rightarrow\{1, \ldots, q\}, G_{t}$ has a $q$-coloring $\gamma$ with $\left.\gamma\right|_{B_{t}}=\gamma_{t}$ without a monochromatic maximal clique in $V_{t} \backslash B_{t}$ if and only if the analogous condition holds for both $G_{s_{1}}$ and $G_{s_{2}}$. Therefore, for all such $\gamma_{t}$, we let $\operatorname{tab}\left[t, \gamma_{t}\right]=1$ if and only if $\operatorname{tab}\left[s_{1}, \gamma_{t}\right]=\operatorname{tab}\left[s_{2}, \gamma_{t}\right]=1$.

Forget Node. Let $t \in V(T)$ be a forget node with child $s$ and let $v$ be the vertex forgotten at $t$, i.e. $B_{s}=B_{t} \cup\{v\}$. A partial solution at node $s$, i.e., a coloring $\gamma_{s}$ of $G_{s}$, may have a monochromatic maximal clique using the vertex $v$, provided that the clique is fully contained in $B_{s}$, while partial solutions at the node $t$ may not. On the other hand, a clique that is maximal inside $B_{S}$ may not be maximal in $G_{s}$. Moreover, as soon as a maximal clique uses a vertex from $V_{s} \backslash B_{S}$ it is not monochromatic in any partial solution at the node $s$, as asserted by the definition of the table entries; provided that $\operatorname{tab}\left[s, \gamma_{s}\right]=1$. We can therefore consult with the oracle $\mathbb{O}_{s}$ to verify if the intersection of any color class with the neighborhood of $v$, together with the vertex $v$, contains a maximal clique in $G_{s}$ (and not just in $G\left[B_{s}\right]$ ). Therefore, for a given coloring $\gamma_{t}: B_{t} \rightarrow\{1, \ldots, q\}$, we can check whether or not there is a partial solution in $G_{t}$ whose restriction to $B_{t}$ is equal to $\gamma_{t}$ as follows. For each color $c \in\{1, \ldots, q\}$, extend $\gamma_{t}$ to a coloring $\gamma_{s}$ of $B_{s}$ by assigning vertex $v$ color $c$. If $\operatorname{tab}\left[s, \gamma_{s}\right]=1$, then we check if the set consisting of $v$ and its neighbors colored $c$ does not contain a maximal clique in $G_{s}$, in which case we can set $\operatorname{tab}\left[t, \gamma_{t}\right]$ to 1 . If there is no color $c$ passing these checks then we know that we can set $\operatorname{tab}\left[t, \gamma_{t}\right]$ to 0 . We summarize this in Algorithm 1.

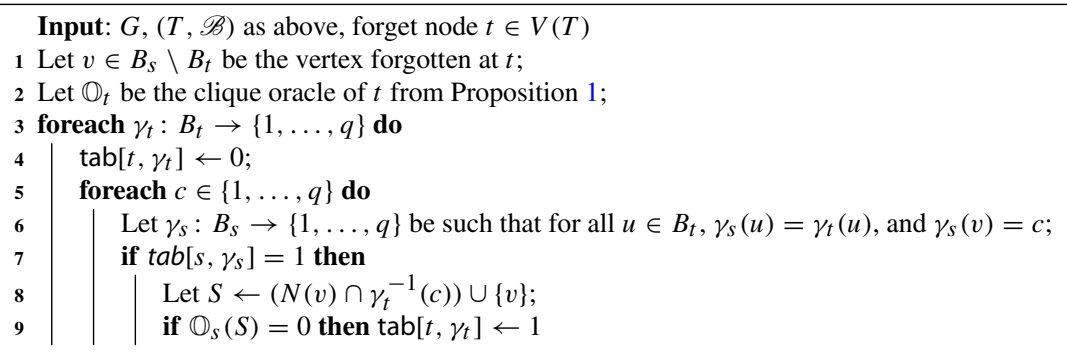

Algorithm 1: Algorithm to compute all table entries at a forget node $t$ with child $s$, assuming all table entries at $s$ have been computed. (Notation: For a set $S \subseteq B_{t}$, $\mathbb{O}_{t}(S)=0$ if and only $S$ contains no maximal clique in $G_{t}$.) 
This completes the description of the algorithm. Correctness follows from the description of the computation of the table entries, by induction on the height of each node. For the runtime, we first take $2^{\mathrm{tw}} \cdot \mathrm{tw}^{\mathscr{O}(1)} \cdot n$ time to construct the clique oracles. At each node, there are at most $q^{\text {tw+1 }}$ table entries to consider, and it is clear that the computation of a table entry at a leaf, introduce, or join node takes constant time. With the clique oracle at hand, computing an entry at a forget node takes time $\mathscr{O}(q)=\mathscr{O}(1)$. Therefore, at each node all table entries can be computed in time $\mathscr{O}\left(q^{\mathrm{tw}}\right)$ and since there are $\mathscr{O}(\mathrm{tw} \cdot n)$ nodes in the tree decomposition, all table entries are computed in time $q^{\mathrm{tw}} \cdot \mathrm{tw}^{\mathscr{O}(1)} \cdot n$, which, since $q \geq 2$, bounds the total runtime of the algorithm. Using standard memoization techniques, the algorithm can also construct a coloring, if one exists.

\subsection{Lower Bound}

In this section we show that the previously presented algorithm is optimal under SETH. In fact, we give lower bounds for much larger parameters than treewidth, which we now define briefly. The feedback vertex set number of a graph $G$ is the size of a smallest set of vertices $S \subseteq V(G)$ such that $G-S$ is a forest. The distance to a linear/caterpillar forest of a graph $G$ is the size of a smallest set $S \subseteq V(G)$ such that $G-S$ is a linear/caterpillar forest.

Our proofs give lower bounds under SETH for the parameters distance to a linear forest (for $q=2$ ), and distance to a caterpillar forest (for $q \geq 3$ ). Note that both paths and caterpillars have pathwidth 1 , and clearly, they do not contain any cycles. Therefore, a lower bound parameterized by the distance to a linear/caterpillar forest implies a lower bound for the parameter pathwidth plus feedback vertex set number. For $q=2$, we give a reduction from $s$ - NOT- ALL-EQUAL SAT ( $s$-NAE-SAT) on $n$ variables. Cygan et al. [13] showed that under SETH, for any $\epsilon>0$, there is some constant $s$ such that $s$-NAE- SAT cannot be solved in time $\mathscr{O}^{\star}\left((2-\epsilon)^{n}\right)$. For all $q \geq 3$, we reduce from $q$ - LIST COLORING, where we are given a graph $G$ and a list for each of its vertices which is a subset of $\{1,2, \ldots, q\}$, and the question is whether $G$ has a proper coloring such that each vertex receives a color from its list. Parameterized by the size $t$ of a deletion set to a linear forest, this problem is known to have no $\mathscr{O}^{\star}\left((q-\epsilon)^{t}\right)$-time algorithms under SETH [22]. Our construction uses the fact that on triangle-free graphs, the proper colorings and the clique colorings coincide, and exploits properties of Mycielski graphs.

We first give the lower bound for the case $q=2$. We would like to remark that Kratochvíl and Tuza [27] gave a reduction from $s$-NOT-ALL-EQUAL SAT to 2CLIQUE COLORING as well, but their reduction does not imply the fine-grained lower bound we aim for here: the resulting graph is at distance $2 n$ to a disjoint union of cliques of constant size (at most $s$ ). This only rules out $\mathscr{O}^{\star}\left((\sqrt{2}-\epsilon)^{t}\right)$-time algorithms parameterized by pathwidth, and does not give any lower bound if the feedback vertex set number is another component of the parameter.

Theorem 3 For any $\epsilon>0$, 2- CLIQUE COLORING parameterized by the distance to a linear forest cannot be solved in time $\mathscr{O}^{\star}\left((2-\epsilon)^{t}\right)$, unless SETH fails. 
Proof We give a reduction from the well-known $s$-NAE- SAT problem, in which we are given a boolean CNF formula $\phi$ whose clauses are of size at most $s$, and the question is whether there is a truth assignment to the variables of $\phi$, such that in each clause, at least one literal evaluates to true and at least one literal evaluates to false.

Let $\phi$ be a boolean CNF formula on $n$ variables $x_{1}, \ldots, x_{n}$ with maximum clause size $s$. We denote by clauses $(\phi)$ the set of clauses of $\phi$ and by $\operatorname{vars}(C)$ the set of variables that appear in the clause $C$ of $\phi$. A clause is called monotone if either all literals are positive or all literals are negated.

Given $\phi$, we construct an instance $G_{\phi}$ for 2- CLIQUE COLORING as follows. For each variable $x_{i}$, we create a vertex $v_{i}$ in $G_{\phi}$. Let $V^{\prime}=\left\{v_{1}, \ldots, v_{n}\right\}$. For each set $S$ of variables, let $V_{S}=\left\{v_{i} \mid x_{i} \in S\right\}$. For each clause $C_{i}$ of $\phi$, we add the following clause gadget to $G_{\phi}$. If $C_{i}$ is monotone, add a path on four vertices to $G_{\phi}$, the end vertices of which are $a_{i}$ and $b_{i}$. Make $N\left(a_{i}\right) \cap V^{\prime}=N\left(b_{i}\right) \cap V^{\prime}=V_{\operatorname{vars}\left(C_{i}\right)}$, and make

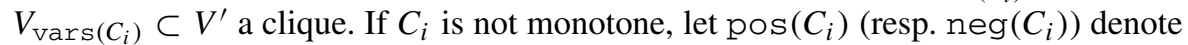
the set of variables with positive (resp. negative) literals in $C_{i}$. Add a path on three vertices to $G_{\phi}$, the end vertices of which are $a_{i}$ and $b_{i}$, make $N\left(a_{i}\right) \cap V^{\prime}=V_{\text {pos }}\left(C_{i}\right)$ and make $V_{\text {pos }\left(C_{i}\right)}$ a clique. Analogously, make $N\left(b_{i}\right) \cap V^{\prime}=V_{\text {neg }\left(C_{i}\right)}$ and make $V_{\text {neg }\left(C_{i}\right)}$ a clique. Finally, add two adjacent vertices $u, v$ to $G_{\phi}$ and make $N[u]=$ $N[v]=\{u, v\} \cup V^{\prime}$. See Fig. 1 .

We will show that $G_{\phi}$ is a yes-instance to 2- Clique COLORING if and only if $\phi$ is a yes-instance to $s$-NAE- SAT. We first make the following observation about the maximal cliques of $G_{\phi}$, which follows directly from the fact that the vertices $u$ and $v$ are complete to $V^{\prime}$.

Observation 2 The vertices $u$ and $v$ belong to every maximal clique of $G_{\phi}\left[V^{\prime} \cup\{u, v\}\right]$.

Claim 1 Let $f: V\left(G_{\phi}\right) \rightarrow\{0,1\}$ be a 2-clique coloring of $G_{\phi}$ and $C_{i}$ be a clause of $\phi$. If $C_{i}$ is monotone, then $f\left(a_{i}\right) \neq f\left(b_{i}\right)$. Otherwise, $f\left(a_{i}\right)=f\left(b_{i}\right)$.

Proof If $C_{i}$ is monotone, $a_{i}$ and $b_{i}$ are the end vertices of a path on four vertices, each edge of which is a maximal clique of $G_{\phi}$. Thus, $f\left(a_{i}\right) \neq f\left(b_{i}\right)$ in any 2-clique

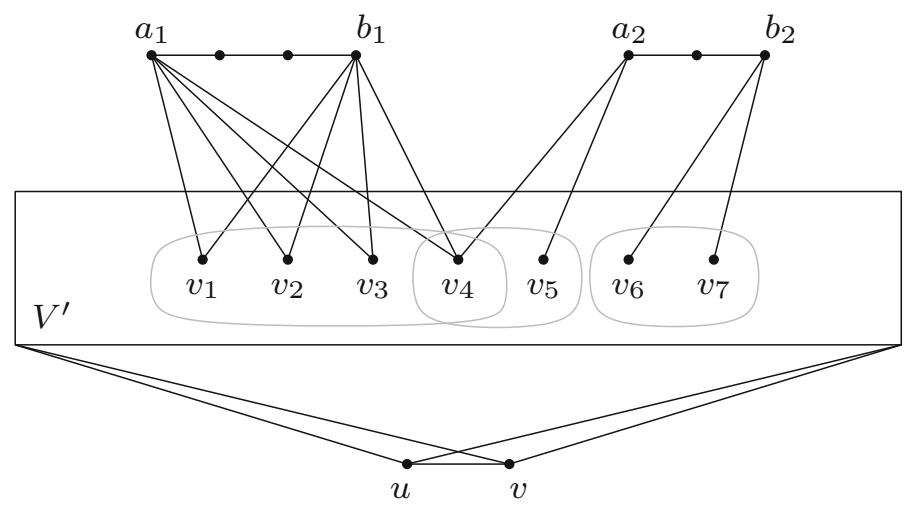

Fig. 1 Depiction of $G_{\phi}$ with two clauses, namely a monotone clause $C_{1}=\neg x_{1} \vee \neg x_{2} \vee \neg x_{3} \vee \neg x_{4}$ and a non-monotone clause $C_{2}=x_{4} \vee x_{5} \vee \neg x_{6} \vee \neg x_{7}$. Note that $G_{\phi}-V^{\prime}$ is a linear forest 
coloring $f$ of $G_{\phi}$. Similarly, if $C_{i}$ is not monotone, $a_{i}$ and $b_{i}$ are the end vertices of a path on three vertices, each edge of which is a maximal clique of $G_{\phi}$. Hence $f\left(a_{i}\right)=f\left(b_{i}\right)$.

Now, suppose $G_{\phi}$ has a 2-clique coloring $f: V\left(G_{\phi}\right) \rightarrow\{0,1\}$. We construct a truth assignment for $\left\{x_{1}, \ldots, x_{n}\right\}$ according to the colors assigned to the vertices of $V^{\prime}$ by $f$. That is, if $f\left(v_{i}\right)=0$, we set $x_{i}$ to false, and if $f\left(v_{i}\right)=1$, we set $x_{i}$ to true. We will now show that this assignment satisfies all clauses of $\phi$. Let $C_{i}$ be a clause of $\phi$. First, assume that $C_{i}$ is monotone. By Claim 1, $f\left(a_{i}\right) \neq f\left(b_{i}\right)$. Since $V_{\operatorname{vars}\left(C_{i}\right)} \cup\left\{a_{i}\right\}$ is a maximal clique of $G_{\phi}$, the vertices of $V_{\operatorname{vars}\left(C_{i}\right)}$ cannot all be colored with $f\left(a_{i}\right)$. Similarly, $V_{\operatorname{vars}\left(C_{i}\right)} \cup\left\{b_{i}\right\}$ is a maximal clique of $G_{\phi}$, the vertices of $V_{\operatorname{vars}\left(C_{i}\right)}$ cannot all be colored with $f\left(b_{i}\right)$. Thus, there exist two vertices $v_{j}, v_{k} \in V_{\operatorname{vars}\left(C_{i}\right)}$ such that $f\left(v_{j}\right) \neq f\left(v_{k}\right)$. Since $C_{i}$ is monotone, this implies that $x_{j}$ and $x_{k}$ are not both evaluated to the same value and therefore $C_{i}$ is satisfied. Now assume $C_{i}$ is not monotone. By

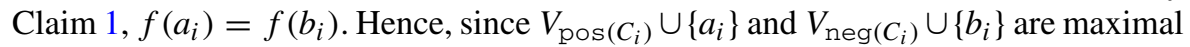
cliques of $G$, there exists $v_{j} \in V_{\mathrm{pos}\left(C_{i}\right)}$ and $v_{k} \in V_{\text {neg }\left(C_{i}\right)}$ such that $f\left(v_{j}\right)=f\left(v_{k}\right)$. This implies that $x_{j}$ and $x_{k}$ are not evaluated to the same value under the proposed assignment and thus $C_{i}$ is satisfied.

For the other direction, assume $\phi$ admits an assignment $\xi$ satisfying all clauses. We construct a clique coloring $f: V\left(G_{\phi}\right) \rightarrow\{0,1\}$ for $G_{\phi}$ in the following way. Color the vertices of $V^{\prime}$ according to the assignment of the variables of $\phi$. That is, if $\xi\left(x_{i}\right)=$ true (resp. $\xi\left(x_{i}\right)=$ false), define $f\left(v_{i}\right)=1$ (resp. $f\left(v_{i}\right)=0$ ). If $C_{i}$ is monotone, let $a_{i} a_{i}^{\prime} b_{i}^{\prime} b_{i}$ be the path on four vertices connecting $a_{i}$ and $b_{i}$ in the clause gadget of $C_{i}$. Define $f\left(a_{i}\right)=f\left(b_{i}^{\prime}\right)=1$ and $f\left(a_{i}^{\prime}\right)=f\left(b_{i}\right)=0$. If $C_{i}$ is not monotone, let $a_{i} a_{i}^{\prime} b_{i}$ be the three vertex path connecting $a_{i}$ and $b_{i}$ in the clause gadget of $C_{i}$. If all the vertices of either $V_{\mathrm{pos}\left(C_{i}\right)}$ or $V_{\text {neg }}\left(C_{i}\right)$ are colored 1 , set $f\left(a_{i}\right)=f\left(b_{i}\right)=0$ and $f\left(a_{i}^{\prime}\right)=1$. Otherwise set $f\left(a_{i}\right)=f\left(b_{i}\right)=1$ and $f\left(a_{i}^{\prime}\right)=0$. Finally, define $f(u)=0$ and $f(v)=1$. To see that this is indeed a 2-clique coloring of $G_{\phi}$, first note that by Observation 2, no maximal clique contained in $G_{\phi}\left[V^{\prime} \cup\{u, v\}\right]$ is monochromatic. Furthermore, since all paths of the clause gadgets are properly colored, no maximal clique contained in $G_{\phi}-\left(V^{\prime} \cup\{u, v\}\right)$ is monochromatic. It remains to show that for each clause $C_{i}$, the maximal cliques $A_{i}=\left\{a_{i}\right\} \cup\left(N\left(a_{i}\right) \cap V^{\prime}\right)$ and $B_{i}=\left\{b_{i}\right\} \cap\left(N\left(b_{i}\right) \cap V^{\prime}\right)$ are not monochromatic. Let $C_{i}$ be a monotone clause. Since $C_{i}$ is satisfied, there exist $x_{j}, x_{k} \in \operatorname{vars}\left(C_{i}\right)$ such that $\xi\left(x_{j}\right) \neq \xi\left(x_{k}\right)$. Hence, $f\left(v_{j}\right) \neq f\left(v_{k}\right)$, which shows that $A_{i}$ and $B_{i}$ are each not monochromatic. If $C_{i}$ is not monotone, by definition the vertices of $A_{i}$ and $B_{i}$ are not all colored 1. Suppose all the vertices of $A_{i}$ are colored 0 . In particular, we have $f\left(a_{i}\right)=f\left(b_{i}\right)=0$. This implies that, by construction, all the vertices of $N\left(b_{i}\right)=V_{\text {neg }}\left(C_{i}\right)$ are colored 1 . However, this is a contradiction with the fact that the clause $C_{i}$ is satisfied, since all its literals are evaluated to false. Hence, $f$ is indeed a 2-clique coloring of $G_{\phi}$.

Finally, note that $G-V^{\prime}$ is a disjoint union of paths of length at most four. Hence, $G$ is at distance $n$ to a linear forest. Therefore, if for some $\epsilon>0,2$ - CLIQUE COLORING parameterized by the distance $t$ to a linear forest can be solved in time $\mathscr{O}^{\star}\left((2-\epsilon)^{t}\right)$, then $s$-NAE- SAT can be solved in time $\mathscr{O}^{\star}\left((2-\epsilon)^{n}\right)$, which would contradict SETH [13]. This concludes the proof. 
We now turn to the case $q \geq 3$. Our reduction is from $q$ - LIST- COLORING parameterized by the distance $t$ to a linear forest, which is known to have no $\mathscr{O}^{\star}\left((q-\epsilon)^{t}\right)$-time algorithms under SETH.

Theorem 4 (Jaffke and Jansen [22]) For any $\epsilon>0$ and any fixed $q \geq 3, q$-LIST COLORING on triangle-free graphs parameterized by the distance $t$ to a linear forest cannot be solved in time $\mathscr{O}^{\star}\left((q-\epsilon)^{t}\right)$, unless SETH fails.

We crucially use the fact that in triangle-free graphs, the proper colorings and the clique colorings coincide, which is both the key and the challenging part of the reduction. In [22], it is not explicitly mentioned that the lower bound from the previous theorem holds on triangle-free graphs, so let us briefly justify this. The reduction presented in [22] is from $s$-SAT on $n$ variables, and given a formula $\phi$, the graph $G_{\phi}$ of the resulting $q$-LIST COLORING instance has the following structure. The truth assignments of the variables of $\phi$ are encoded as colorings of a set of vertices $V^{\prime}$ that are independent in $G_{\phi}$, and for each clause $C$ in $\phi$ and each coloring of some subset $V_{C} \subseteq V^{\prime}$ that corresponds to a truth assignment $\mu$ that does not satisfy $C$, there is a path $P_{\mu}$ in $G$ that cannot be properly list colored if and only if the coloring $\mu$ appears on $V_{C}$. This is ensured by connecting $P_{\mu}$ to $V_{C}$ via a matching, which does not introduce triangles. Since each edge of $G_{\phi}$ is either on such a path or part of one of such matching, there are no triangles in $G_{\phi}$.

Now, Theorem 4 already gives a lower bound, under SETH, for the list-version of $q$-CLIQUE COLORING parameterized by the distance to a linear forest. To obtain the desired lower bound (without lists), we need to simulate the lists without introducing triangles. For proper colorings, there is a standard way to simulate lists that is frequently used in such reductions (e.g., [22,28]): We add a clique on vertex set $[q]$ to the input graph $G$, and for each $i \in[q]$ and $v \in V(G)$, we add an edge between $i$ and $v$ if color $i$ does not appear on the list of $v$. Then, $G$ has a coloring respecting the given lists if and only if the resulting graph has a proper coloring with $q$ colors. In our setting, however, this clearly does not work, since the previous step introduces triangles of two kinds:

1. Between any triple of vertices in the $q$-clique.

2. Between vertices of $G$ and the $q$-clique.

To avoid triangles of type 1 , we replace the $q$-clique by a gadget $H_{q}$ consisting of several Mycielski graphs that are connected to each other in such a way that we can identify a set of $q$ vertices that receive pairwise distinct colors in any proper coloring. ${ }^{3}$ These $q$ vertices can then be used in the same way as the vertices of the $q$-clique mentioned above. Since Mycielski graphs are triangle-free, and by the way we connect them, $H_{q}$ is a triangle-free graph. To avoid triangles of type 2, we do not connect the remaining vertices to $H_{q}$ directly, but instead we add a middle layer of vertices that we connect to $H_{q}$ in such a way that only one given color can appear on each vertex in any proper coloring. This way we propagate the forcing of colors while avoiding triangles of type 2 . The latter step is also the reason why our lower bound

\footnotetext{
3 We would like to remark that also Marx [30] used Mycielski graphs and their properties in hardness proofs for the CLIQUE COLORING problem.
} 
only holds when parameterized by the distance to a caterpillar forest and not by the distance to a linear forest: after removing the modulator of the $q$-LIST-COLORING instance and the gadget $H_{q}$, the remaining graph consists of the linear forest from the $q$ - LIST- COLORING instance and the vertices of the middle layer; together they form a caterpillar forest.

Theorem 5 For any $\epsilon>0$ and any fixed $q \geq 3$, $q$-CLIQUE COLORING parameterized by the distance $t$ to a caterpillar forest cannot be solved in time $\mathscr{O}^{\star}\left((q-\epsilon)^{t}\right)$, unless SETH fails.

Proof We give a reduction from $q$-LIST COLORING on triangle-free graphs parameterized by distance to linear forest. In this proof we use the phrases " $q$-colorable" as short for "can be properly colored with at most $q$ colors", and " $q$-coloring" as short for "a proper coloring with at most $q$ colors". To construct our instance of $q$ - CLIQUE COLORING, we will first describe the construction of a color selection gadget, and then describe how this gadget is attached to the rest of the graph. The description of the color selection gadget makes use of the famous Mycielski graphs. For completeness, we briefly describe how Mycielski graphs are recursively constructed and some of their useful properties. For every $p \geq 2$, the Mycielski graph $M_{p}$ is a trianglefree graph with chromatic number $p$. For $p=2$, we define $M_{2}=K_{2}$. For $p \geq 3$, the graph $M_{p}$ is obtained from $M_{p-1}$ as follows. Let $V\left(M_{p-1}\right)=\left\{v_{1}, \ldots, v_{n}\right\}$. Then $V\left(M_{p}\right)=V\left(M_{p-1}\right) \cup\left\{u_{1}, \ldots, u_{n}, w\right\}$. The vertices of $V\left(M_{p-1}\right)$ induce a copy of $M_{p-1}$ in $M_{p}$, each $u_{i}$ is adjacent to all the neighbors of $v_{i}$ in $M_{p-1}$ and $N(w)=\left\{u_{1}, \ldots, u_{n}\right\}$. Hence, $\left|V\left(M_{p}\right)\right|=3 \cdot 2^{p-2}-1$. Moreover, it is known that $M_{p}$ is edge-critical, that is, the deletion of any edge of $M_{p}$ leads to a $(p-1)$-colorable graph (see for instance [5,29]). For our construction, we will use the graph $M_{p}^{\prime}$, obtained from $M_{p}$ by the deletion of an arbitrary edge $x y$. The following observation follows directly from the fact that $M_{p}$ is edge-critical.

Observation 3 Let $M_{p}^{\prime}$ be the graph obtained from $M_{p}$ by the deletion of an edge $x y$. Then, $M_{p}^{\prime}$ is $(p-1)$-colorable, and in any $(p-1)$-coloring of $M_{p}^{\prime}$, the vertices $x$ and $y$ receive the same color.

Color selection gadget. We construct a gadget $H_{q}$ in the following way. Consider $q$ disjoint copies of $M_{q+1}^{\prime}$. For $1 \leq i \leq q$, let $x_{i} y_{i}$ be the edge removed from $M_{q+1}$ in order to obtain the $i$ th copy of $M_{q+1}^{\prime}$. For each $i$, add $q-1$ false twins to $y_{i}$. We denote these vertices by $y_{i j}$, with $1 \leq j \leq q, j \neq i$. Then delete the vertex $y_{i}$, for every $i$. Note that this graph is still $q$-colorable and, by Observation 3, in every such $q$-coloring, for each $i$, the vertices $x_{i}$ and $y_{i j}$, for all $j \neq i$, receive the same color. Now we add $\left(\begin{array}{l}q \\ 2\end{array}\right)$ edges to connect the copies of $M_{q+1}^{\prime}$ : for $1 \leq i<j \leq q$, add the edge $y_{i j} y_{j i}$ to $H_{q}$. Note that $H_{q}$ remains triangle-free after the addition of these edges, since for all $1 \leq i<j \leq q, N\left(y_{i j}\right) \cap N\left(y_{j i}\right)=\emptyset$. We will need the following property of the $q$-colorings of $H_{q}$.

Claim 2 The graph $H_{q}$ is $q$-colorable. Moreover, in any $q$-coloring $\phi$ of $H_{q}, \phi\left(x_{i}\right) \neq$ $\phi\left(x_{j}\right)$ for all $1 \leq i<j \leq q$. 
Proof Suppose for a contradiction that there exists a $q$-coloring of $H_{q}$ such that $\phi\left(x_{i}\right)=\phi\left(x_{j}\right)$, for some $i \neq j$. By Observation 3, we know that $\phi\left(x_{i}\right)=\phi\left(y_{i j}\right)$. Similarly, $\phi\left(x_{j}\right)=\phi\left(y_{j i}\right)$. This implies that $\phi\left(y_{i j}\right)=\phi\left(y_{j i}\right)$, which is a contradiction, since $y_{i j}$ and $y_{j i}$ are adjacent by construction. To see that a $q$-coloring indeed exists for $H_{q}$, first note that, by Observation 3, each copy of $M_{q+1}^{\prime}$ has a $q$-coloring in which $x_{i}$ and $y_{i}$ are assigned the same color. We can then permute the colors within a copy to obtain a proper coloring of that copy in which $x_{i}$ and $y_{i}$ receive color $i$. To complete the coloring, assign color $i$ to every $y_{i j}$ that is a false twin of $y_{i}$. This yields a proper $q$-coloring of $H_{q}$.

We are now ready to describe the construction of our instance $G^{\prime}$ to $q$-CLIQUE COLORING. Let $(G, L)$ be an instance of $q$-LIST COLORING on triangle-free graphs that is at distance $t$ from a linear forest. We construct $G^{\prime}$ as follows. Add a copy of $G$ and a copy of $H_{q}$ to $G^{\prime}$. We denote by $V^{\prime}$ the set of vertices corresponding to $V(G)$ in $G^{\prime}$. For each $v \in V^{\prime}$, add $q-|L(v)|$ vertices adjacent to $v$. We denote these vertices by $\left\{v_{j} \mid j \notin L(v)\right\}$. Finally, make $v_{j}$ adjacent to all the vertices of $\left\{x_{\ell} \mid \ell \neq j\right\}$. See Fig. 2.

Note that $G^{\prime}$ is triangle-free since $H_{q}$ and $G$ are triangle-free, and $N\left(v_{j}\right) \cap V^{\prime}=\{v\}$ and $N\left(v_{j}\right) \cap V\left(H_{q}\right)$ is an independent set. Furthermore, let $S \subseteq V(G)$ be a set such that $G-S$ is a linear forest and $|S|=t$. Then $S \cup V\left(H_{q}\right)$ is such that each connected component of $G^{\prime}-\left(S \cup V\left(H_{q}\right)\right)$ is a caterpillar and $\left|S \cup V\left(H_{q}\right)\right|=t+q\left(3 \cdot 2^{q-1}+\right.$ $q-3)=t+\mathscr{O}(1)$, since $q$ is a constant.

We will show that $(G, L)$ is a yes-instance to $q$-LIST COLORING if and only if $G^{\prime}$ is a yes-instance to $q$-CLIQUE COLORING. Note that since $G^{\prime}$ is a triangle-free graph, every clique coloring of $G^{\prime}$ is a proper coloring of it as well. First, suppose $(G, L)$ is a yes-instance to $q$-LIST COLORING and let $\phi$ be a $q$-list coloring for $G$. We give a $q$-coloring $\phi^{\prime}$ for $G^{\prime}$ in the following way. If $v \in V^{\prime}$, make $\phi^{\prime}(v)=\phi(v)$. For each

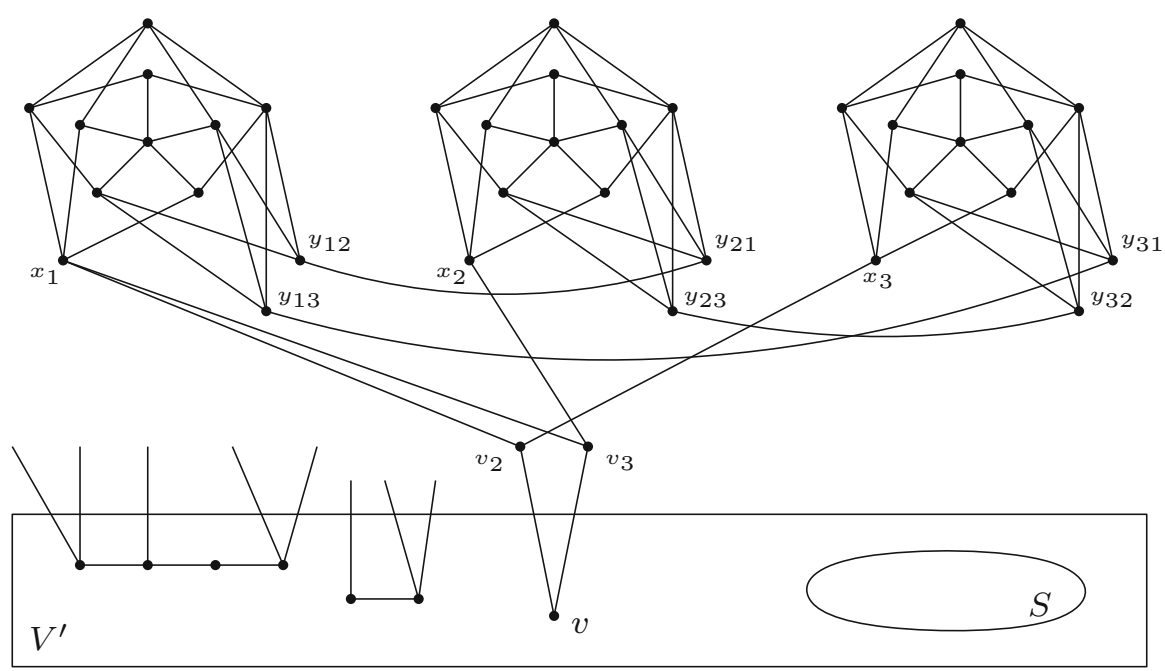

Fig. 2 In this instance, $q=3$ and $L(v)=\{1\}$. Note that $G^{\prime}-\left(S \cup V\left(H_{q}\right)\right)$ is a caterpillar forest 
$v_{j} \in N(v)$, make $\phi^{\prime}\left(v_{j}\right)=j$. Note that since $j \notin L(v)$, we have that $\phi^{\prime}(v) \neq \phi\left(v_{j}\right)$. Finally, consider a proper $q$-coloring of $H_{q}$. By Claim 2, the vertices $x_{1}, \ldots, x_{q}$ were assigned pairwise distinct colors. Without loss of generality, we can assume $x_{i}$ received color $i$. Extend $\phi$ to the remaining vertices of $G^{\prime}$ according to this coloring of $H_{q}$. This leads to a proper $q$-coloring of $G^{\prime}$, since $\phi\left(v_{j}\right)=j$ and $v_{j}$ is not adjacent to $x_{j}$.

Now assume $G^{\prime}$ admits a $q$-clique coloring $\phi$. We will show that $\left.\phi\right|_{V^{\prime}}$ is a $q$-list coloring for $(G, L)$. Since $G^{\prime}$ is triangle-free, it is clear that $\left.\phi\right|_{V^{\prime}}$ is a proper coloring of $G$. It remains to show it satisfies the constraints imposed by the lists. By Claim 2, we can again assume that $\phi\left(x_{i}\right)=i$, for every $i$. For every $v \in V^{\prime}$, since $\left\{x_{\ell} \mid \ell \neq j\right\} \subset N\left(v_{j}\right)$, we necessarily have $\phi\left(v_{j}\right)=j$. Finally, since for every $c \notin L(v)$ there is a neighbor of $v$ that is colored $c$ (namely $v_{c}$ ), we conclude that $\phi(v) \in L(v)$.

Now, suppose that $q$-CLIQUE COLORING admits an algorithm running in time $\mathscr{O}^{\star}\left((q-\epsilon)^{t^{\prime}}\right)$, for some $\epsilon>0$, where $t^{\prime}$ is the distance of the input graph to a caterpillar forest. Then, we can solve $q$ - LIST- COLORING paramterized by the distance $t$ to a linear forest by applying the above reduction, giving a $q$-CLIQUE COLORING instance at distance $t+\mathscr{O}(1)$ to a caterpillar forest, and solving the resulting $q$-CLIQUE COLORING instance. Correctness is argued in the previous paragraphs, and the runtime of the resulting algorithm is $\mathscr{O}^{\star}\left((q-\epsilon)^{t+\mathscr{O}(1)}\right)=\mathscr{O}^{\star}\left((q-\epsilon)^{t}\right)$, contradicting SETH by Theorem 4 .

Since the instance of $q$-CLIQUE COLORING constructed in the proof of Theorem 5 is a triangle-free graph, we obtain the following corollary.

Corollary 1 For any $\epsilon>0$ and any fixed $q \geq 3, q$-COLORING on triangle-free graphs parameterized by the distance $t$ to a caterpillar forest cannot be solved in time $\mathscr{O}^{\star}((q-$ $\left.\epsilon)^{t}\right)$, unless SETH fails.

\section{Parameterized by Clique-Width}

In this section, we give an XP-time algorithm for CLIQUE COLORING parameterized by clique-width, more precisely, parameterized by the equivalent measure module-width. We provide an algorithm that given an $n$-vertex graph $G$ with one of its rooted branch decompositions of module-width $w$ and an integer $k$, decides whether $G$ has a clique coloring with $k$ colors in time $k^{f(w)} \cdot n$, where $f(w)=2^{2^{\mathscr{\theta}(w)}}$. Before we describe the algorithm, we give a high level outline of its main ideas, and where the double exponential dependence on $w$ in the degree of the polynomial comes from.

The algorithm is bottom-up dynamic programming along the given branch decomposition of the input graph. Let $t$ be some node in the branch decomposition. To keep the number of table entries bounded by something that is XP in the module-width, we have to find a way to group color classes into types whose number is upper bounded by a function of $w$ alone. The intention is that two color classes of the same type are interchangeable with respect to the underlying coloring being completable to a valid clique coloring of the whole graph. Partial solutions (colorings of the subgraph $G_{t}$ ) can then be described by remembering, for each type, how many color classes of that type there are. If the number of types is $f(w)$ for some function $f$, this gives 
an upper bound of $k^{f(w)}$ on the number of table entries at each node of the branch decomposition.

Let us discuss what kind of information goes into the definition of a type. Since the final coloring of $G$ has to avoid monochromatic maximal cliques, we maintain information about cliques in $G_{t}$ that are or may become monochromatic maximal cliques in some extension of the coloring at hand. A natural attempt would be to consider and describe maximal cliques in $G_{t}$ by their intersection patterns with the equivalence classes of $\sim_{t}$. However, it is not sufficient to consider only maximal cliques in $G_{t}$; given a maximal clique $X$ in $G_{t}$, it may happen that in $\overline{V_{t}}$ there is a vertex $v$ that is adjacent to a strict subset $Y \subset X$ of that clique, forming a maximal clique with $Y$ - which does not fully contain $X$ - in a supergraph of $G_{t}$. Considering the equivalence classes of $\sim_{t}$, this implies that the equivalence classes containing $Y$ and the ones containing $X \backslash Y$ are disjoint. We therefore consider cliques $X$ that are maximal in the subgraph induced by the equivalence classes containing vertices of $X$. We call such cliques $X$ eqc-maximal, and observe that with a little extra information, we can keep track of the forming and disintegrating of eqc-maximal cliques along the branch decomposition. If an eqc-maximal clique is fully contained in some set of vertices (/color class) $C$, then we call it potentially bad for $C$. A potentially bad clique is described via its profile, which consists of the intersection pattern with the equivalence classes of $\sim_{t}$, and some extra information. At each node, there are at most $2^{\mathscr{O}(w)}$ profiles.

Equipped with this definition, we can define the notion of a $t$-type of a color class $C$, which is simply the subset of profiles at $t$, such that $G_{t}$ contains a potentially bad clique with that $C$-profile. It immediately follows that the number of $t$-types is $2^{2^{\mathscr{O}(w)}}$. Now, colorings $\mathscr{C}_{t}$ of $G_{t}$ are described by their $t$-signature, which records how many color classes of each type $\mathscr{C}_{t}$ has. There are at most $k^{f(w)}$ many $t$-signatures, where $f(w)=2^{2^{\mathscr{O}(w)}}$, and this essentially bounds the runtime of the resulting algorithm to $n \cdot k^{f(w)}=n^{\mathscr{O}(f(w))}$.

At the root node $\mathfrak{r} \in V(T)$, there is only one equivalence class, namely $V_{\mathfrak{r}}=V(G)$, and if in a coloring, there is a clique that is potentially bad for some color class, then it is indeed a monochromatic maximal clique. Therefore, at the root node, we only have to check whether there is a coloring all of whose color classes have no potentially bad cliques.

\subsection{Potentially Bad Cliques}

We now introduce the main concept used to describe color classes in partial solutions of our algorithms, namely potentially bad cliques. These are cliques that are monochromatic in some subgraph induced by a set of equivalence classes.

Definition 5 (Potentially bad clique) Let $G$ be a graph with rooted branch decomposition $(T, \mathscr{L})$ and let $t \in V(T)$. A clique $X$ in $G_{t}$ is called eqc-maximal (in $G_{t}$ ) if it is maximal in $G_{t}\left[V\left(\operatorname{eqc}_{t}(X)\right)\right]$. Let $C \subseteq V_{t}$ and let $X$ be a clique in $G_{t}$. Then, $X$ is called potentially bad for $C$ (in $G_{t}$ ), if $X$ is eqc-maximal in $G_{t}$ and $X \subseteq C$. 


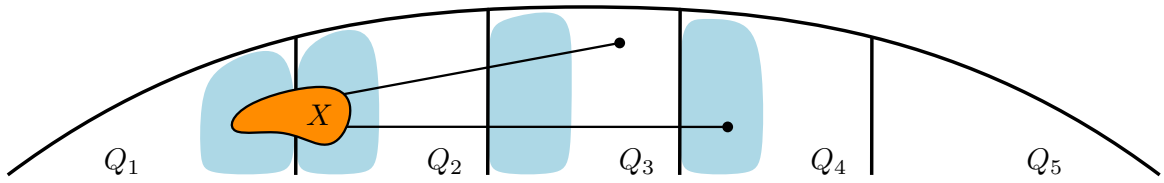

Fig. 3 Illustration of the $C$-profile of a clique $X$ that is potentially bad for a color class $C$, depicted as the shaded areas within the equivalence classes. In this case, we have that $\pi(X \mid C)=\left(\left\{Q_{1}, Q_{2}\right\},\left\{Q_{3}, Q_{4}\right\}\right)$ (Color figure online)

Naturally, it is not feasible to keep track of all potentially bad cliques. We therefore capture the most vital information about potentially bad cliques in the following notion of a profile. For our algorithm, it is only important to know for a color class whether or not it has some potentially bad clique with a given profile, rather than how many, or what its vertices are. This is key to reduce the amount of information we need to store about partial solutions.

There are two components of a profile of a potentially bad clique $X$; the first one is the set of equivalence classes $\mathscr{Q}$ containing its vertices, and the second one consists of the equivalence classes $P \notin \mathscr{Q}$ that have a vertex that is complete to $X$. This is because, at a later stage, $P$ may be merged with an equivalence class containing vertices of $X$ (via the bubbles), in which case $X$ is no longer potentially bad. We illustrate the following definition in Fig. 3.

Definition 6 (Profile) Let $G$ be a graph with rooted branch decomposition $(T, \mathscr{L})$ and let $t \in V(T)$. Let $C \subseteq V_{t}$ and let $X$ be a clique in $G_{t}$ that is potentially bad for $C$. The $C$-profile of $X$ is a pair of subsets of $V_{t} / \sim_{t}, \pi(X \mid C):=(\mathscr{Q}, \mathscr{P})$, where

$$
\mathscr{Q}=\operatorname{eqc}_{t}(X) \text { and } \mathscr{P}=\left\{P \in \overline{\operatorname{eqc}}_{t}(X) \mid \exists v \in P: X \subseteq N(v)\right\} .
$$

We call the set of all pairs of disjoint subsets of $V_{t} / \sim_{t}$, where the first coordinate is nonempty, the profiles at $t$, formally, $\Pi_{t}:=\left\{(\mathscr{Q}, \mathscr{P}) \mid \mathscr{Q}, \mathscr{P} \subseteq V_{t} / \sim_{t}: \mathscr{Q} \neq\right.$ $\emptyset \wedge \mathscr{Q} \cap \mathscr{P}=\emptyset\}$.

Observation 4 Let $(T, \mathscr{L})$ be a rooted branch decomposition. For each $t \in V(T)$, there are at most $2^{\mathscr{O}(w)}$ profiles at $t$, where $w=m w(T, \mathscr{L})$.

Let $t \in V(T) \backslash \mathrm{L}(T)$ be an internal node with children $r$ and $s$ and operator $\left(H_{t}, \eta_{r}, \eta_{s}\right)$, and let $\pi_{r} \in \Pi_{r}$ and $\pi_{s} \in \Pi_{s}$ be a pair of profiles. We are now working towards a notion that precisely captures when and how a potentially bad clique in $G_{r}$ for some $C_{r} \subseteq V_{r}$ with $C_{r}$-profile $\pi_{r}$ can be merged with a potentially bad clique in $G_{s}$ for some $C_{s} \subseteq V_{s}$ with $C_{s}$-profile $\pi_{s}$ to obtain a potentially bad clique for $C_{r} \cup C_{s}$ in $G_{t}$. As it turns out, if this is possible, then the profile of the resulting clique only depends on $\pi_{r}, \pi_{s}$, and the operator of $t$. Note that for now, we focus on the case when the cliques in $G_{r}$ and $G_{s}$ are both nonempty, and we discuss the case when one of them is empty below.

Before we proceed with this description, we need to introduce some more concepts. We illustrate all of the following concepts in Fig. 4. For a set of equivalence classes $\mathscr{S} \subseteq V_{r} / \sim_{r} \cup V_{s} / \sim_{s}$, its bubble buddies at $t$, denoted by bb $b_{t}(\mathscr{S})$, are the equivalence 


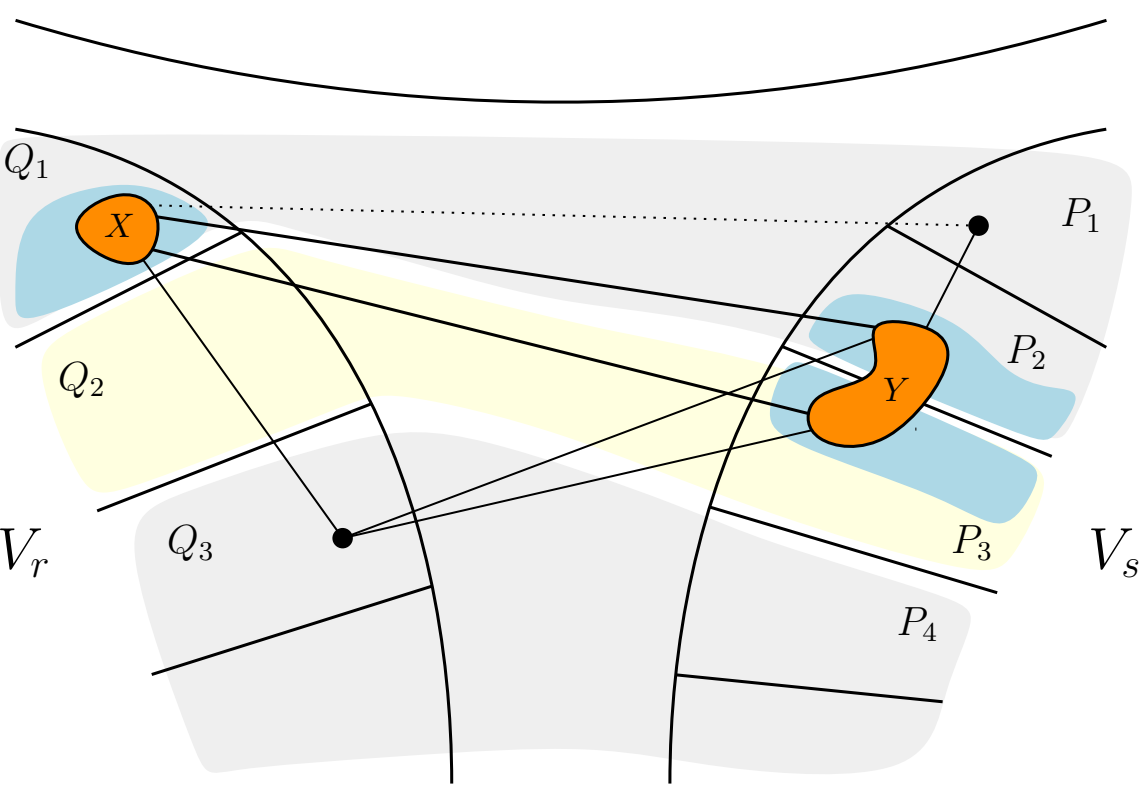

Fig. 4 Merging a potentially bad clique $X$ in $G_{r}$ with a potentially bad clique $Y$ in $G_{S}$ to obtain a potentially bad clique in $G_{t}$. The color class at hand is depicted in blue and the gray and yellow areas show the (three) bubbles. Note that the equivalence classes $P_{1}$ and $Q_{2}$ are bubble buddies of eqc $c_{r}(X)$ and eqc $c_{s}(Y)$. Moreover, the types of $X$ and $Y$ are compatible, since $\left\{Q_{1}, P_{2}, P_{3}\right\}$ is a maximal biclique in $H_{t}\left[\left\{Q_{1}, P_{1}, P_{2}, P_{3}\right\}\right]$. The dotted line between the vertex in $P_{1}$ and $Q_{1}$ shows that there is no edge between the vertices in $P_{1}$ and $Q_{1}$. Observe that if these edges were present, then $X$ and $Y$ would not be compatible, since $\left\{Q_{1}, P_{2}, P_{3}\right\}$ would no longer be a maximal biclique in $H_{t}\left[\left\{Q_{1}, P_{1}, P_{2}, P_{3}\right\}\right]$. Finally, note that the equivalence class of $\sim_{t}$ corresponding to the bubble containing $Q_{3}$ will have a vertex that is complete to the potentially bad clique $X \cup Y$ (Color figure online)

classes of $V_{r} / \sim_{r} \cup V_{s} / \sim_{s}$ that are in the same bubble as some equivalence class in $\mathscr{S}$ :

$$
\operatorname{bb}_{t}(\mathscr{S}):=\bigcup_{p \in\{r, s\}}\left\{Q_{p} \in V_{p} / \sim_{p} \mid \eta_{p}\left(Q_{p}\right) \in \eta_{p}\left(\mathscr{S} \cap V_{p} / \sim_{p}\right)\right\}
$$

We say that $\pi_{r}=\left(\mathscr{Q}_{r}, \mathscr{P}_{r}\right)$ and $\pi_{s}=\left(\mathscr{Q}_{s}, \mathscr{P}_{s}\right)$ are compatible, if $\mathscr{Q}_{r} \cup \mathscr{Q}_{s}$ is a maximal biclique in

$$
H_{t}^{\prime}\left(\pi_{r}, \pi_{s}\right):=H_{t}\left[\left(\mathscr{Q}_{r} \cup \mathscr{Q}_{s}\right) \cup\left(\left(\mathscr{P}_{r} \cup \mathscr{P}_{s}\right) \cap \mathrm{bb}_{t}\left(\mathscr{Q}_{r} \cup \mathscr{Q}_{s}\right)\right)\right]
$$

As we show below, the notion of compatibility precisely captures the 'merging behavior' of potentially bad cliques. Moreover, for $\pi_{r}$ and $\pi_{s}$ compatible, we can immediately construct the profile of the resulting potentially bad clique: the merge profile of $\pi_{r}$ and $\pi_{s}$ is the profile $\mu\left(\pi_{r}, \pi_{s}\right)=\left(\mathscr{Q}_{t}, \mathscr{P}_{t}\right)$ such that

- $\mathscr{Q}_{t}=\eta_{r}\left(\mathscr{Q}_{r}\right) \cup \eta_{s}\left(\mathscr{Q}_{s}\right)$ and

- $\mathscr{P}_{t}=\bigcup_{\{o, p\}=\{r, s\}}\left\{\eta_{p}\left(Q_{p}\right) \mid Q_{p} \in \mathscr{P}_{p} \backslash \mathrm{bb}_{t}\left(\mathscr{Q}_{r} \cup \mathscr{Q}_{s}\right): \mathscr{Q}_{o} \subseteq N_{H_{t}}\left(Q_{p}\right)\right\}$.

Lemma 2 Let $t \in V(T) \backslash \mathrm{L}(T)$ be an internal node with children $r$ and $s$ and operator $\left(H_{t}, \eta_{r}, \eta_{s}\right)$. For all $p \in\{r, s\}$, let $C_{p} \subseteq V_{p}$, let $X_{p}$ be a clique in $G_{p}$ that is 
potentially bad for $C_{p}$, and let $\pi_{p}:=\pi\left(X_{p} \mid C_{p}\right)=\left(\mathscr{Q}_{p}, \mathscr{P}_{p}\right)$. If $\pi_{r}$ and $\pi_{s}$ are compatible, then $X_{t}:=X_{r} \cup X_{s}$ is a clique that is potentially bad for $C_{t}:=C_{r} \cup C_{s}$, and $\pi\left(X_{t} \mid C_{t}\right)=\mu\left(\pi_{r}, \pi_{s}\right)$.

Proof We first argue that $X_{t}$ is a clique. Since $X_{r}$ and $X_{s}$ are cliques, we only have to show that for each $v_{r} \in X_{r}$ and $v_{s} \in X_{s}, v_{r} v_{s} \in E\left(G_{t}\right)$. In other words, if $Q_{r}$ is the equivalence class of $\sim_{r}$ containing $v_{r}$, and $Q_{s}$ is the equivalence class of $\sim_{s}$ containing $v_{s}$, then $Q_{r} Q_{s} \in E\left(H_{t}\right)$. Now, $Q_{r} \in \operatorname{eqc}_{r}\left(X_{r}\right)=\mathscr{Q}_{r}$ and $Q_{s} \in \operatorname{eqc}_{s}\left(X_{s}\right)=\mathscr{Q}_{s}$, and since $\pi_{r}$ and $\pi_{s}$ are compatible, we have that $\mathscr{Q}_{r} \cup \mathscr{Q}_{s}$ is a biclique in $H_{t}$, therefore $Q_{r} Q_{s} \in E\left(H_{t}\right)$.

Next, we show that $X_{t}$ is potentially bad for $C_{t}$. Since $X_{r}$ and $X_{s}$ are potentially bad for $C_{r}$ and $C_{s}$, respectively, we have that $X_{r} \subseteq C_{r}$ and $X_{s} \subseteq C_{s}$, and therefore $X_{t}=X_{r} \cup X_{s} \subseteq C_{r} \cup C_{s}=C_{t}$. It remains to show that $X_{t}$ is eqc-maximal. Suppose not, and let $y \in V\left(\operatorname{eqc}_{t}\left(X_{t}\right)\right)$ be a vertex that is complete to $X_{t}$. First, we know that $y \notin V\left(\operatorname{eqc}_{r}\left(X_{r}\right) \cup \operatorname{eqc}_{s}\left(X_{s}\right)\right)$, for if $y \in V\left(\operatorname{eqc}_{p}\left(X_{p}\right)\right)$ for some $p \in\{r, s\}$, then $X_{p}$ is not eqc-maximal, contradicting $X_{p}$ being potentially bad for $C_{p}$. On the other hand, we have that eqc $c_{t}\left(X_{t}\right)=\operatorname{bb}_{t}\left(\operatorname{eqc}_{r}\left(X_{r}\right) \cup \operatorname{eqc}_{s}\left(X_{s}\right)\right)=\operatorname{bb}_{t}\left(\mathscr{Q}_{r} \cup \mathscr{Q}_{s}\right)$. We may assume that for some $p \in\{r, s\}$, the vertex $y$ is contained in some $Q_{p} \in$ $\mathrm{bb}_{t}\left(\mathscr{Q}_{r} \cup \mathscr{Q}_{s}\right) \backslash\left(\mathscr{Q}_{r} \cup \mathscr{Q}_{s}\right)$. Assume up to renaming that $p=r$. Since $y$ is complete to $X_{t}$, we have that $y$ is complete to $X_{r}$, and therefore $Q_{r} \in \mathscr{P}_{r}$. In other words, $Q_{r}$ is contained in the graph $H_{t}^{\prime}\left(\pi_{r}, \pi_{s}\right)$ as described in Eq. (1). Moreover, since $y$ is complete to $X_{s}$, we have that $Q_{r}$ is complete to eqc ${ }_{s}\left(X_{s}\right)=\mathscr{Q}_{s}$. This implies that $\left\{Q_{r}\right\} \cup \mathscr{Q}_{r} \cup \mathscr{Q}_{s}$ is a biclique in $H_{t}^{\prime}\left(\pi_{r}, \pi_{s}\right)$, contradicting $\pi_{r}$ and $\pi_{s}$ being compatible.

To conclude the proof, we need to show that $\pi\left(X_{t} \mid C_{t}\right)=\mu\left(\pi_{r}, \pi_{s}\right)$. Let $\mu\left(\pi_{r}, \pi_{s}\right)=\left(\mathscr{Q}_{t}, \mathscr{P}_{t}\right)$. We first show that eqc $c_{t}\left(X_{t}\right)=\mathscr{Q}_{t}$.

To see that $\mathscr{Q}_{t}=\eta_{r}\left(\mathscr{Q}_{r}\right) \cup \eta_{s}\left(\mathscr{Q}_{s}\right) \subseteq \operatorname{eqc}_{t}\left(X_{t}\right)$, we observe that for all $Q_{p} \in \mathscr{Q}_{p}$, there is an $x \in X_{p} \cap Q_{p}$. This means that $x \in \eta_{p}\left(Q_{p}\right)$, therefore $X_{t} \cap \eta_{p}\left(Q_{p}\right) \neq \emptyset$ and $\eta_{p}\left(Q_{p}\right) \in \operatorname{eqc}_{t}\left(X_{t}\right)$. The other inclusion can be argued similarly.

Now suppose that $Q_{t} \in \mathscr{P}_{t}$. Then, for some $\{o, p\}=\{r, s\}, Q_{t}=\eta_{p}\left(Q_{p}\right)$ for some $Q_{p} \in \mathscr{P}_{p} \backslash \mathrm{bb}_{t}\left(\mathscr{Q}_{r} \cup \mathscr{Q}_{s}\right)$ with $\mathscr{Q}_{o} \subseteq N_{H_{t}}\left(Q_{p}\right)$. In other words, there is a vertex $v \in Q_{p}$ that is complete to $X_{t}$, and $\eta_{p}\left(Q_{p}\right) \notin$ eqc $_{t}\left(X_{t}\right)$. According to the definition of a profile, $Q_{t}=\eta_{p}\left(Q_{p}\right)$ is contained in the second coordinate of $\pi_{t}$. The other inclusion can be shown similarly.

Now we show the other direction, i.e. that if we have a potentially bad clique for some $C_{t} \subseteq V_{t}$ in $G_{t}$, then its restrictions to $V_{r}$ and $V_{s}$ necessarily also form potentially bad cliques for the restriction of $C_{t}$ to $V_{r}$ and $V_{s}$ in $G_{r}$ and $G_{s}$, respectively. Furthermore, in that case, the profiles of the resulting cliques are compatible.

Lemma 3 Let $t \in V(T) \backslash \mathrm{L}(T)$ be an internal node with children $r$ and $s$ and operator $\left(H_{t}, \eta_{r}, \eta_{s}\right)$. Let $C_{t} \subseteq V_{t}$, and let $X_{t}$ be a clique in $G_{t}$ that is potentially bad for $C_{t}$. For all $p \in\{r, s\}$, let $X_{p}:=X_{t} \cap V_{p}$ and $C_{p}:=C_{t} \cap V_{p}$. Suppose that for all $p \in\{r, s\}, X_{p} \neq \emptyset$. Then, for all $p \in\{r, s\}, X_{p}$ is a potentially bad clique for $C_{p}$, and $\pi_{r}:=\pi\left(X_{r} \mid C_{r}\right)$ and $\pi_{s}:=\pi\left(X_{s} \mid C_{s}\right)$ are compatible.

Proof Since $X_{t}$ is a potentially bad clique for $C_{t}$, we have that $X_{t} \subseteq C_{t}$, and so for $p \in\{r, s\}, X_{p} \subseteq C_{p}$. It remains to show that $X_{p}$ is eqc-maximal for all $p \in\{r, s\}$. 
Up to renaming, it suffices to show that $X_{r}$ is eqc-maximal. Suppose not and let $y \in \operatorname{eqc}_{r}\left(X_{r}\right)$ be a vertex that is complete to $X_{r}$. Since $X_{t}$ is a clique in $G_{t}$, we have that eqc ${ }_{r}\left(X_{r}\right) \cup$ eqc $_{s}\left(X_{s}\right)$ is a biclique in $H_{t}$. Therefore, $y$ is also complete to $X_{s}$ and therefore to $X_{t}$. Clearly, $y \in \operatorname{eqc}_{t}\left(X_{t}\right)$, and we have a contradiction with $X_{t}$ being eqc-maximal.

What remains to be shown is that $\pi_{r}=\left(\mathscr{Q}_{r}, \mathscr{P}_{r}\right)$ and $\pi_{s}=\left(\mathscr{Q}_{s}, \mathscr{P}_{s}\right)$ are compatible. We have already argued that $\mathscr{Q}_{r} \cup \mathscr{Q}_{s}=\operatorname{eqc}_{r}\left(X_{r}\right) \cup \operatorname{eqc} c_{s}\left(X_{s}\right)$ is a biclique in $H_{t}$; we have to show that $\mathscr{Q}_{r} \cup \mathscr{Q}_{s}$ is a maximal biclique in $H_{t}^{\prime}:=H_{t}^{\prime}\left(\pi_{r}, \pi_{s}\right)$ as defined in Eq. (1). Clearly, $\mathscr{Q}_{r} \cup \mathscr{Q}_{s} \subseteq V\left(H_{t}^{\prime}\right)$, so suppose that $\mathscr{Q}_{r} \cup \mathscr{Q}_{s}$ is not a maximal biclique in $H_{t}^{\prime}$. This means that for some $p \in\{r, s\}$, there is some $Q_{p} \in \mathscr{P}_{p} \cap \mathrm{bb}_{t}\left(\mathscr{Q}_{r} \cup \mathscr{Q}_{s}\right)$ such that $\left\{Q_{p}\right\} \cup \mathscr{Q}_{r} \cup \mathscr{Q}_{s}$ is a biclique in $H_{t}^{\prime}$. In that case, there is a vertex $y \in Q_{p}$ that is complete to $X_{t}$ (since $Q_{p} \in \mathscr{P}_{p}$ and $\left\{Q_{p}\right\} \cup \mathscr{Q}_{r} \cup \mathscr{Q}_{s}$ is a biclique), and $y \in V\left(\operatorname{eqc}_{t}\left(X_{t}\right)\right)\left(\right.$ since $Q_{p} \in \operatorname{bb}_{t}\left(\mathscr{Q}_{r} \cup \mathscr{Q}_{s}\right)$ ); we obtained a contradiction with $X_{t}$ being eqc-maximal.

As mentioned above, we treat the case when a clique $X_{p}$ in one of the children $p \in\{r, s\}$ remains potentially bad in $G_{t}$ separately. This is because in that case, the notion of a maximal biclique in $H_{t}^{\prime}$ as defined in Equation (1) does not hold up very naturally. We formulate the analogous requirements for this case here, and we skip some of the details.

Let $t \in V(T) \backslash \mathrm{L}(T)$ be an internal node with children $r$ and $s$ and operator $\left(H_{t}, \eta_{r}, \eta_{s}\right)$. Let $\pi_{r} \in \Pi_{r}$. We say that $\pi_{r}=\left(\mathscr{Q}_{r}, \mathscr{P}_{r}\right)$ is liftable if

- There is no $Q_{s} \in \mathrm{bb}_{t}\left(\mathscr{Q}_{r}\right)$ that is complete to $\mathscr{Q}_{r}$ in $H_{t}$, and

$-\operatorname{bb}_{t}\left(\mathscr{Q}_{r}\right) \cap \mathscr{P}_{r}=\emptyset$.

The lift profile of $\pi_{r}$, denoted by $\lambda\left(\pi_{r}\right)$, is constructed as the merge profile of $\pi_{r}$ with the empty set; i.e. we take $\left(\mathscr{Q}_{s}, \mathscr{P}_{s}\right)=\left(\emptyset, V_{s} / \sim_{s}\right)$ and apply the definition given above, meaning $\lambda\left(\pi_{r}\right)=\mu\left(\pi_{r},\left(\emptyset, V_{s} / \sim_{s}\right)\right)$.

Lemma 4 Let $t \in V(T) \backslash \mathrm{L}(T)$ be an internal node with children $r$ and $s$. Let $C_{r} \subseteq V_{r}$, $C_{s} \subseteq V_{s}$, let $X_{r}$ be a clique in $G_{r}$, and let $\pi_{r}:=\pi\left(X_{r} \mid C_{r}\right)$. Then, $X_{r}$ is a potentially bad clique for $C_{r} \cup C_{s}$ in $G_{t}$ if and only if $X_{r}$ is a potentially bad clique for $C_{r}$ in $G_{r}$ and $\pi_{r}$ is liftable, in which case $\pi_{t}\left(X_{r} \mid C_{r} \cup C_{s}\right)=\lambda\left(\pi_{r}\right)$.

Proof The proof can be done with very similar arguments to those given above and is therefore omitted. One only needs to observe that the notion of 'liftable' modulates the notion of a profile being compatible with the profile of an empty set.

\subsection{The Type of a Color Class}

We now describe the $t$-type of a color class $C$, which is the subset of profiles at $t$ such that there is a clique in $G_{t}$ that is potentially bad for $C$, with that $C$-profile. For our algorithm, two color classes with the same type will be interchangeable, therefore we only have to remember the number of color classes of each type. 
Definition 7 ( $t$-Type) Let $G$ be a graph with rooted branch decomposition $(T, \mathscr{L})$, and let $t \in V(T)$. For a set $C \subseteq V_{t}$, the $t$-type of $C$, denoted by $\gamma_{t}(C)$ is

$$
\begin{aligned}
\gamma_{t}(C):=\left\{\pi_{t} \in \Pi_{t} \mid\right. & \exists \text { clique } X \text { in } G_{t} \text { which is potentially bad for } C \\
& \text { and } \left.\pi(X \mid C)=\pi_{t}\right\} .
\end{aligned}
$$

With slight abuse of notation, we call the set $\Gamma_{t}=2^{\Pi_{t}}$ of all subsets of profiles at $t$ the $t$-types.

Since for each $t \in V(T),\left|\Pi_{t}\right| \leq 2^{\mathscr{O}(w)}$ by Observation 4, the number of $t$-types can be upper bounded as follows.

Observation 5 Let $(T, \mathscr{L})$ be a rooted branch decomposition, and let $t \in V(T)$. There are at most $2^{2^{\mathscr{O}(w)}}$ many $t$-types, where $w:=\operatorname{mw}(T, \mathscr{L})$.

In our algorithm we want to be able to determine the $t$-type of the union of a color class in $G_{r}$ and a color class in $G_{s}$. This is done via the following notion of a merge type, which is based on the notion of merge and lift profiles given in the previous section.

Definition 8 (Merge type) Let $G$ be a graph with rooted branch decomposition $(T, \mathscr{L}$ ), let $t \in V(T) \backslash \mathrm{L}(T)$ with children $r$ and $s$. For a pair of an $r$-type $\gamma_{r} \in \Gamma_{r}$ and an $s$-type $\gamma_{s} \in \Gamma_{s}$, the merge type of $\gamma_{r}$ and $\gamma_{s}$, denoted by $\mu\left(\gamma_{r}, \gamma_{s}\right)$, is the $t$-type obtained as follows.

$$
\begin{aligned}
\mu\left(\gamma_{r}, \gamma_{s}\right):= & \left\{\mu\left(\pi_{r}, \pi_{s}\right) \mid \pi_{r} \in \gamma_{r}, \pi_{s} \in \gamma_{s}, \text { where } \pi_{r} \text { and } \pi_{s} \text { are compatible }\right\} \\
& \bigcup_{p \in\{r, s\}}\left\{\lambda\left(\pi_{p}\right) \mid \pi_{p} \in \gamma_{p}, \text { where } \pi_{p} \text { is liftable }\right\}
\end{aligned}
$$

Lemma 5 Let $G$ be a graph with rooted branch decomposition $(T, \mathscr{L})$, let $t \in$ $V(T) \backslash \mathrm{L}(T)$ with children $r$ and $s$. Let $C_{r} \subseteq V_{r}$ and $C_{s} \subseteq V_{s}$. Then, $\gamma_{t}\left(C_{r} \cup C_{s}\right)=$ $\mu\left(\gamma_{r}\left(C_{r}\right), \gamma_{s}\left(C_{s}\right)\right)$.

Proof Let $C_{t}:=C_{r} \cup C_{s}$. For one inclusion, let $\pi_{t} \in \gamma_{t}\left(C_{t}\right)$. Then, there is a clique $X_{t}$ in $G_{t}$ that is potentially bad for $C_{t}$ whose $C_{t}$-profile is $\pi_{t}$. If for all $p \in\{r, s\}$, $X_{p}:=X_{t} \cap V_{p} \neq \emptyset$, then by Lemma 3, we know that for all $p \in\{r, s\}, X_{p}$ is a potentially bad clique for $C_{p}:=C_{t} \cap V_{p}$, therefore $\pi_{p}:=\pi\left(X_{p} \mid C_{p}\right) \in \gamma_{p}\left(C_{p}\right)$. Moreover, the lemma asserts that $\pi_{r}$ and $\pi_{s}$ are compatible, so by construction, we can conclude that $\pi_{t}=\mu\left(\pi_{r}, \pi_{s}\right) \in \mu\left(\gamma_{r}\left(C_{r}\right), \gamma_{s}\left(C_{s}\right)\right)$. On the other hand, if for some $p \in\{r, s\}, X_{t} \subseteq V_{p}$, then by Lemma $4, X_{t}$ is a potentially bad clique for $C_{p}$, so $\pi_{p}:=\pi_{p}\left(X_{t} \mid C_{p}\right) \in \gamma\left(C_{p}\right)$. The lemma also asserts that $\pi_{p}$ is liftable and that $\lambda\left(\pi_{r}\right)=\pi_{t}$, in which case we also have that $\pi_{t} \in \mu\left(\gamma_{r}\left(C_{r}\right), \gamma_{s}\left(C_{s}\right)\right)$. We have argued that $\gamma_{t}\left(C_{t}\right) \subseteq \mu\left(\gamma_{r}\left(C_{r}\right), \gamma_{s}\left(C_{s}\right)\right)$.

For the other inclusion, suppose that $\pi_{t} \in \mu\left(\gamma_{r}\left(C_{r}\right), \gamma_{s}\left(C_{s}\right)\right)$. Then, either there is a pair of profiles $\pi_{r} \in \gamma_{r}\left(C_{r}\right), \pi_{s} \in \gamma_{s}\left(C_{s}\right)$ such that $\pi_{r}$ and $\pi_{s}$ are compatible and $\pi_{t}=\mu\left(\pi_{r}, \pi_{s}\right)$ or for some $p \in\{r, s\}$, there is a profile $\pi_{p} \in \gamma_{p}\left(C_{p}\right)$ that is liftable and $\pi_{t}=\lambda\left(\pi_{p}\right)$. In the former case, we can use Lemma 2 to conclude that $\pi_{t} \in \gamma_{t}\left(C_{t}\right)$, and in the latter case, we have that $\pi_{t} \in \gamma_{t}\left(C_{t}\right)$ by Lemma 4 . This shows that $\mu\left(\gamma_{r}\left(C_{r}\right), \gamma_{s}\left(C_{s}\right)\right) \subseteq \gamma_{t}\left(C_{t}\right)$ which concludes the proof. 


\subsection{The Algorithm}

We are now ready to describe the algorithm. As alluded to above, partial solutions at a node $t$, i.e. colorings of $G_{t}$, are described via the notion of a $t$-signature which records the number of color classes of each type in a coloring. If two colorings have the same $t$-signature, then they are interchangeable as far as our algorithm is concerned. We show that this information suffices to solve the problem in a bottom-up dynamic programming fashion.

Definition 9 ( $t$-Signature) Let $k$ be a positive integer. Let $G$ be a graph with rooted branch decomposition $(T, \mathscr{L})$, let $t \in V(T)$, and let $\mathscr{C}=\left(C_{1}, \ldots, C_{k}\right)$ be a $k$-coloring of $G_{t}$. Then, $\sigma_{\mathscr{C}}: \Gamma_{t} \rightarrow\{0,1, \ldots, k\}$ where

$$
\forall \gamma_{t} \in \Gamma_{t}: \sigma_{\mathscr{C}}\left(\gamma_{t}\right):=\left|\left\{i \in\{1, \ldots, k\} \mid \gamma_{t}\left(C_{i}\right)=\gamma_{t}\right\}\right|,
$$

is called the $t$-signature of $\mathscr{C}$. The set of $t$-signatures is defined as:

$$
\operatorname{sig}_{t}:=\left\{\sigma_{t}: \Gamma_{t} \rightarrow\{0,1, \ldots, k\} \mid \sum_{\gamma_{t} \in \Gamma_{t}} \sigma_{t}\left(\gamma_{t}\right)=k\right\}
$$

The following bound on the number of $t$-signatures immediately follows from Observation 5 , stating that the number of $t$-types is upper bounded by $2^{2^{\mathscr{O}(w)}}$.

Observation 6 Let $(T, \mathscr{L})$ be a rooted branch decomposition of an $n$-vertex graph, and

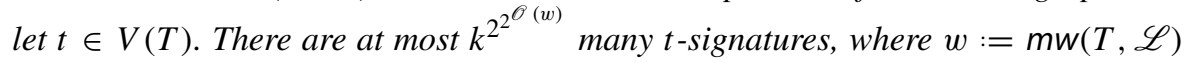
and $k$ is the number of colors.

Definition of the Table Entries For each $t \in V(T)$ and $\sigma_{t} \in \operatorname{sig}_{t}$, we let $\operatorname{tab}\left[t, \sigma_{t}\right]=1$ if and only if there is a $k$-coloring $\mathscr{C}$ of $G_{t}$ such that $\sigma_{\mathscr{C}}=\sigma_{t}$.

We now show that the information stored at the table entries suffices to determine whether or not our input is a YES-instance; that is, after filling all the table entries, we can read off the solution to the problem at the root node.

Lemma 6 Let $G$ be a graph with rooted branch decomposition $(T, \mathscr{L})$, and let $\mathfrak{r}$ be the root of $T$. $G$ has a clique coloring with $k$ colors if and only if $\operatorname{tab}\left[\mathfrak{r}, \sigma^{\star}\right]=1$, where $\sigma^{\star}$ is the $\mathfrak{r}$-signature for which $\sigma^{\star}(\emptyset)=k$.

Proof The lemma immediately follows from two facts. First, since $\sigma^{\star}(\emptyset)=k$, we have that $\sigma^{\star}\left(\gamma_{\mathfrak{r}}\right)=0$ for any other $\mathfrak{r}$-type $\gamma_{\mathfrak{r}} \neq \emptyset$. Second, that for each set $C \subseteq V_{\mathfrak{r}}=V(G)$, the set of potentially bad cliques for $C$ is precisely the set of maximal cliques that are fully contained in $C$, i.e. it is the set of monochromatic maximal cliques in the corresponding coloring that are contained in $C$.

We first describe how to compute the table entries at the leaves, by brute-force.

Leaves of $\boldsymbol{T}$ Let $t \in \mathrm{L}(T)$ be a leaf node in $T$ and let $v \in V(G)$ be the vertex such that $\mathscr{L}(v)=t$. We show how to compute the table entries $\operatorname{tab}[t, \cdot]$. Note that $G_{t}=(\{v\}, \emptyset)$, 


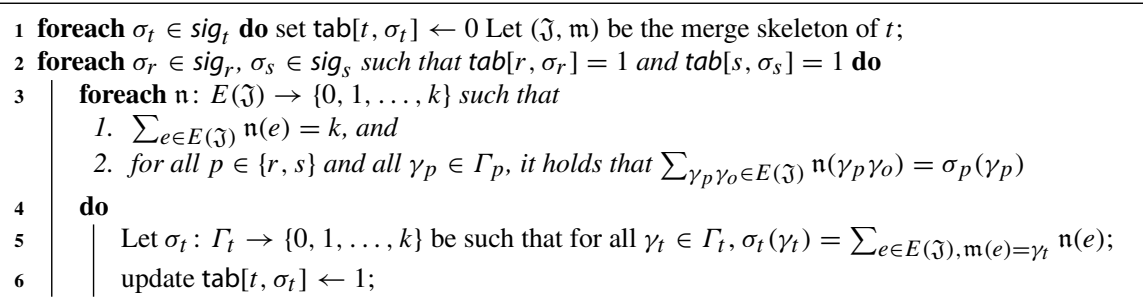

Algorithm 2: Algorithm to set the table entries at an internal node $t \in V(T) \backslash \mathrm{L}(T)$ with children $r$ and $s$, assuming the table entries at $r$ and $s$ have been computed.

and that $\{v\}$ is the only equivalence class of $\sim_{t}$. To describe the types of color classes of $G_{t}$, observe that the only eqc-maximal clique in $G_{t}$ is $\{v\}=: X_{v}$, which is potentially bad for $C_{v}:=\{v\}=X_{v}$. In that case, we have that $\pi_{v}:=\pi\left(X_{v} \mid C_{v}\right)=(\{v\}, \emptyset)$, and the type of color class $C_{v}$ is $\left\{\pi_{v}\right\}$. The type of the remaining $k-1$ color classes is $\emptyset$, since they are all empty. Therefore, for each $t$-signature $\sigma_{t}$, we set $\operatorname{tab}\left[t, \sigma_{t}\right]:=1$ if and only if $\sigma_{t}\left(\left\{\pi_{v}\right\}\right)=1$ and $\sigma_{t}(\emptyset)=k-1$.

Next, we move on to the computation of the table entries at internal nodes of the branch decomposition. To describe this part of the algorithm, we borrow the following notion of a merge skeleton from [23]. ${ }^{4}$

Definition 10 (Merge skeleton) Let $G$ be a graph and $(T, \mathscr{L})$ one of its rooted branch decompositions. Let $t \in V(T) \backslash \mathrm{L}(T)$ with children $r$ and $s$. The merge skeleton of $r$ and $s$ is a pair $(\mathfrak{J}, \mathfrak{m})$, where $\mathfrak{J}$ is a complete bipartite graph and $\mathfrak{m}: E(\mathfrak{J}) \rightarrow \Gamma_{t}$ is an edge-labeling of $\mathfrak{J}$ with

$-V(\mathfrak{J})=\Gamma_{r} \cup \Gamma_{s}$, and

- for all $\gamma_{r} \in \Gamma_{r}, \gamma_{s} \in \Gamma_{s}, \mathfrak{m}\left(\gamma_{r} \gamma_{s}\right)=\mu\left(\gamma_{r}, \gamma_{s}\right)$.

Internal nodes of $\boldsymbol{T}$ Let $t \in V(T) \backslash \mathrm{L}(T)$ be an internal node with children $r$ and $s$. We discuss how to compute the table entries at $t$, assuming the table entries at $r$ and $s$ have been computed. Each coloring of $G_{t}$ can be obtained from a coloring of $G_{r}$ and a coloring of $G_{s}$, by merging pairs of color classes. Therefore, for each pair $\sigma_{r} \in \operatorname{sig}_{r}, \sigma_{s} \in \operatorname{sig}_{s}$ such that $\operatorname{tab}\left[r, \sigma_{r}\right]=1$ and $\operatorname{tab}\left[s, \sigma_{s}\right]=1$, we do the following. We enumerate all labelings of the edge set of the merge skeleton with numbers from $\{0,1, \ldots, k\}$, with the following interpretation. If an edge $\gamma_{r} \gamma_{s}$ has label $j$, then it means that $j$ color classes of $r$-type $\gamma_{r}$ will be merged with $j$ color classes of $s$-type $\gamma_{s}$; this gives $j$ color classes of $t$-type $\mu\left(\gamma_{r}, \gamma_{s}\right)=\mathfrak{m}\left(\gamma_{r} \gamma_{s}\right)$. Each such labeling that respects the number of color classes available of each type will produce a coloring of $G_{t}$ with some signature $\sigma_{t}$, which can then be read off the edge labeling. For all such $\sigma_{t}$, we set $\operatorname{tab}\left[t, \sigma_{t}\right]=1$. We give the formal details in Algorithm 2 .

We now prove the correctness of the algorithm.

Lemma 7 Let $G$ be a graph and $(T, \mathscr{L})$ one of its rooted branch decompositions, and let $t \in V(T)$. The above algorithm computes the table entries tab $[t, \cdot]$ correctly, i.e.

\footnotetext{
4 Note that in [23], the graph structure of the bipartite graph plays a role, in that there is only edges between compatible types. In the present setting, there is no notion of compatibility of color class types which is why the bipartite graph of the merge skeleton is always complete.
} 
for each $\sigma_{t} \in \operatorname{sig}_{t}$, it sets $\operatorname{tab}\left[t, \sigma_{t}\right]=1$ if and only if $G_{t}$ has a $k$-coloring $\mathscr{C}$ with $\sigma_{\mathscr{C}}=\sigma_{t}$.

Proof The proof is by induction on the height of $t$. In the base case, when $t$ is a leaf, it is straightforward to verify correctness.

Now suppose that $t \in V(T) \backslash \mathrm{L}(T)$ is an internal node with children $r$ and $s$, and let $(\mathfrak{J}, \mathfrak{m})$ be the merge skeleton at $t$. Suppose for some $t$-signature $\sigma_{t} \in \operatorname{sig}_{t}$, the algorithm set $\operatorname{tab}\left[t, \sigma_{t}\right]=1$. Then, there is some $r$-signature $\sigma_{r}$ and some $s$-signature $\sigma_{s}$ such that $\operatorname{tab}\left[r, \sigma_{r}\right]=1, \operatorname{tab}\left[s, \sigma_{s}\right]=1$, and there is a map $\mathfrak{n}: E(\mathfrak{J}) \rightarrow\{0,1, \ldots, k\}$ satisfying the conditions of lines 3 and 5 in Algorithm 2. By induction, there is a $k$-coloring $\mathscr{C}_{r}$ of $G_{r}$ whose $r$-signature is $\sigma_{r}$, and a $k$-coloring of $\mathscr{C}_{s}$ of $G_{s}$ whose $s$-signature is $\sigma_{s}$. We construct the desired coloring $\mathscr{C}_{t}$ of $G_{t}$ whose $t$-signature is $\sigma_{t}$ as follows: For each pair of an $r$-type $\gamma_{r}$ and an $s$-type $\gamma_{s}$, we take $\mathfrak{n}\left(\gamma_{r} \gamma_{s}\right)$ pairs of a color class $C_{r}$ of $r$-type $\sigma_{r}$ and a color class $C_{s}$ of $s$-type $C_{s}$, and for each such pair, we add $C_{r} \cup C_{s}$ as a color class to $\mathscr{C}_{t}$. By Lemma 5, the $t$-type of $C_{r} \cup C_{s}$ is $\mu\left(\gamma_{r}, \gamma_{s}\right)=\mathfrak{m}\left(\gamma_{r} \gamma_{s}\right)$. The condition in line 3 ensures that each color class of $\mathscr{C}_{r}$ and each color class of $\mathscr{C}_{s}$ is used precisely once to create a color class of $\mathscr{C}_{t}$ (which also implies that $\mathscr{C}_{t}$ has $k$ color classes), and the condition in line 5 ensures that the $t$-signature of $\mathscr{C}_{t}$ is indeed $\sigma_{t}$.

For the other direction, suppose that there is a $k$-coloring $\mathscr{C}_{t}$ of $G_{t}$ with $t$-signature $\sigma_{t}$. We construct a pair of a coloring $\mathscr{C}_{r}$ of $G_{r}$ and a coloring of $\mathscr{C}_{s}$ of $G_{s}$, together with their signatures $\sigma_{r}$ and $\sigma_{s}$, respectively, and a map $\mathfrak{n}: E(\mathfrak{J}) \rightarrow\{0,1, \ldots, k\}$. Initially, for all $p \in\{r, s\}$, we let $\mathscr{C}_{p}=\emptyset$, and for all $\gamma_{p} \in \Gamma_{p}, \sigma_{p}\left(\gamma_{p}\right):=0$. Moreover, we let $\mathfrak{n}(e):=0$ for all $e \in E(\mathfrak{J})$.

For each color class $C_{t} \in \mathscr{C}_{t}$, we add $C_{r}:=C_{t} \cap V_{r}$ to $\mathscr{C}_{r}$ and $C_{s}:=C_{t} \cap V_{s}$ to $\mathscr{C}_{s}$. Let $\gamma_{t}$ be the $t$-type of $C_{t}$. By Lemma 5, $C_{r}$ has some $r$-type $\gamma_{r}$ and $C_{s}$ has some $s$-type $\gamma_{s}$ such that $\gamma_{t}$ is the merge type $\mu\left(\gamma_{r}, \gamma_{s}\right)$ of $\gamma_{r}$ and $\gamma_{s}$. We increase the values of $\sigma_{r}\left(\gamma_{r}\right)$ and $\sigma_{s}\left(\gamma_{s}\right)$ by 1 , since we added one more color class of $r$-type $\gamma_{r}$ to $\mathscr{C}_{r}$, and one more color class of $s$-type $\gamma_{s}$ to $\mathscr{C}_{s}$. Additionally, we add 1 to the value of $\mathfrak{n}\left(\gamma_{r} \gamma_{s}\right)$, since $C_{t}$ is a color class of $t$-type $\mu\left(\gamma_{r}, \gamma_{s}\right)=\mathfrak{m}\left(\gamma_{r} \gamma_{s}\right)$ obtained from merging $C_{r}$ (a color class of $r$-type $\gamma_{r}$ ) with $C_{s}$ (a color class of $s$-type $\gamma_{s}$ ).

After doing this for all color classes of $\mathscr{C}_{t}$, we have that $\mathscr{C}_{r}$ is a $k$-coloring with $r$ signature $\sigma_{r}$, and that $\mathscr{C}_{s}$ is a $k$-coloring with $s$-signature $\sigma_{s}$. By induction, $\operatorname{tab}\left[r, \sigma_{r}\right]=$ 1 and $\operatorname{tab}\left[s, \sigma_{s}\right]=1$. It remains to argue that $\mathfrak{n}$ satisfies the conditions expressed in lines 3 and 5 in Algorithm 2. The first item of line 3 is clearly satisfied, since we increased $\left|\mathscr{C}_{t}\right|=k$ values of $\mathfrak{n}$ by 1 in the above process. The second item holds since we increased the value of some $\sigma_{p}\left(\gamma_{p}\right)$ by 1 if and only if we increased the value of an edge $e$ incident with $\gamma_{p}$ in $\mathfrak{J}$ by 1 . To see that for each $\gamma_{t}, \sigma_{t}\left(\gamma_{t}\right)=$ $\sum_{e \in E(\mathfrak{J}), \mathfrak{m}(e)=\gamma_{t}} \mathfrak{n}(e)$, observe that we identified for each color class of type $\gamma_{t}$, the occurrence of $\gamma_{t}$ as a merge type of a pair of an $r$-type and an $s$-type, and therefore a label of some edge $e \in E(\mathfrak{J})$, and increased $\mathfrak{n}(e)$ by 1 in such a case. We can conclude that $\sigma_{t}$ can be obtained as shown in line 5 of Algorithm 2, and so the algorithm set $\operatorname{tab}\left[t, \sigma_{t}\right]=1$.

To wrap up, it remains to argue the runtime of the algorithm. Suppose we are given a graph $G$ with rooted branch decomposition $(T, \mathscr{L})$ and let $w:=\operatorname{mw}(T, \mathscr{L})$. By Observation 6, there are at most $k^{2^{2^{\mathscr{O}(w)}}}$ table entries at each node of $T$. The entries 
of leaf nodes can clearly be computed in constant time. Now let $t \in V(T) \backslash \mathrm{L}(T)$ be an internal node with children $r$ and $s$. To compute all table entries at $t$, we execute Algorithm 2. In the worst case, it loops over each pair of an $r$-signature and an $s$ signature, and given such a pair, it enumerates all labelings of the edges of the merge skeleton $\mathfrak{J}$ with numbers from $\{0,1, \ldots, k\}$ (such that all entries sum up to $k$ ). We have that $|E(\mathfrak{J})|=\left|\Gamma_{r}\right| \cdot\left|\Gamma_{s}\right|=\left(2^{2^{\mathscr{O}(w)}}\right)^{2}=2^{2^{\mathscr{O}(w)}}$ (see Observation 5), therefore the number of labelings to consider is upper bounded by $k^{2^{2^{\mathscr{O}(w)}}}$. The runtime of Algorithm 2 can therefore be upper bounded by

$$
\left(k^{2^{2^{\mathscr{O}}(w)}}\right)^{2} \cdot k^{2^{2^{\mathscr{O}}(w)}}=k^{2^{2^{\mathscr{O}}(w)}},
$$

and since $|V(T)|=\mathscr{O}(n)$, the runtime of the whole procedure is $k^{2^{\mathscr{O}^{(}(w)}} \cdot n$. Correctness is proved in Lemmas 7, and reflem:ccol:root asserts that the solution to the problem can be read off the table entries at the root, once computed. Using standard memoization techniques, we can modify the above algorithm so that it returns a coloring if one exists. Lastly, we observe that we may assume that $k<n$. For if $k \geq n$, then the input instance is a trivial YES-instance: we can simply assign each vertex of the input graph $G$ a distinct color; this clearly results in a clique coloring of $G$. We have the following theorem.

Theorem 6 There is an algorithm that given an n-vertex graph $G$ together with one of its rooted branch decompositions $(T, \mathscr{L})$ and a positive integer $k$, decides whether $G$ has a clique coloring with $k$ colors in time $k^{2^{2^{\mathscr{O}(w)}}} \cdot n \leq n^{2^{2^{\mathscr{O}}(w)}}$, where $w:=$ $m w(T, \mathscr{L})$. If such a coloring exists, the algorithm can construct it.

\section{Conclusion}

In this work, we considered structural parameterizations of the CLIQUE COLORING problem by two of the most commonly used width measures of graphs: treewidth and clique-width. We showed that for fixed number of colors $q \geq 2, q$ - CLIQUE COLORING can be solved in time $\mathscr{O}^{\star}\left(q^{\mathrm{tw}}\right)$, where tw denotes the width of a given tree decomposition of the input graph, and that under SETH, there is no such algorithm running in time $\mathscr{O}^{\star}\left((q-\epsilon)^{\mathrm{tw}}\right)$, for any $\epsilon>0$. Regarding the clique-width parameterization, we gave a $k^{2^{2^{\mathscr{O}}(\mathrm{cw})}} \cdot n$ time algorithm, where $k$ is the requested number of colors and the input graph is given together with a clique-width cw-expression. We would like to end this work by recalling and explicitly stating the open questions from the introduction that are raised by the clique-width based algorithm. First, we would be interested in the coarse parameterized complexity of this problem.

Open Problem 1 Is CLIQUE COLORING parameterized by clique-width W[1]-hard?

Arguably the most promising route to an FPT-algorithm for CLIQUE COLORING parameterized by clique-width (if it exists) is via a proof that the number of colors 
that is needed in any clique coloring is upper bounded in terms of some function of the clique-width of a graph.

Open Problem 2 Is there a function $g: \mathbb{N} \rightarrow \mathbb{N}$ such that each graph $G$ can be clique colored with at most $g(\mathrm{cW})$ colors, where $\mathrm{cw}$ denotes the clique-width of $G$ ?

Next, it would be interesting to see if the triple-exponential dependence on cw of our algorithm can be avoided - both in the case when the number of colors is unbounded and when the number of colors is bounded.

Open Problem 3 Is there an algorithm for CLIQUE COLORING running in time $n^{2^{2^{o(\mathrm{cw})}}}$, or for fixed $q \geq 2$, an algorithm for $q$-CLIQUE COLORING running in time $q^{2^{2^{o(\mathrm{cw})}}}$. $n^{\mathscr{O}(1)}$, when the input graph is given together with a clique-width cw-expression, or would any such algorithm violate ETH?

Acknowledgements We would like to thank the anonymous reviewers for comments that helped improving the presentation of this paper.

Funding Open access funding provided by University of Bergen (incl Haukeland University Hospital)

\section{Declarations}

Conflict of interest The authors declare that they have no conflict of interest.

Open Access This article is licensed under a Creative Commons Attribution 4.0 International License, which permits use, sharing, adaptation, distribution and reproduction in any medium or format, as long as you give appropriate credit to the original author(s) and the source, provide a link to the Creative Commons licence, and indicate if changes were made. The images or other third party material in this article are included in the article's Creative Commons licence, unless indicated otherwise in a credit line to the material. If material is not included in the article's Creative Commons licence and your intended use is not permitted by statutory regulation or exceeds the permitted use, you will need to obtain permission directly from the copyright holder. To view a copy of this licence, visit http://creativecommons.org/licenses/by/4.0/.

\section{References}

1. Andreae, T., Schughart, M., Tuza, Z.: Clique-transversal sets of line graphs and complements of line graphs. Discrete Math. 88(1), 11-20 (1991)

2. Bacsó, G., Gravier, S., Gyárfás, A., Preissmann, M., Sebo, A.: Coloring the maximal cliques of graphs. SIAM J. Discrete Math. 17(3), 361-376 (2004)

3. Bacsó, G., Tuza, Z.: Clique-transversal sets and weak 2-colorings in graphs of small maximum degree. Discrete Math. Theor. Comput. Sci. 11(2), 15-24 (2009)

4. Björklund, A., Husfeldt, T., Kaski, P., Koivisto, M.: Fourier meets Möbius: fast subset convolution. In: Johnson, D.S., Feige, U. (eds.) Proceedings of the 39th Annual ACM Symposium on Theory of Computing (STOC 2007), pp. 67-74. ACM (2007)

5. Bondy, J.A., Murty, U.S.R.: Graph Theory. Springer, New York (2008)

6. Campos, C.N., Dantas, S., de Mello, C.P.: Colouring clique-hypergraphs of circulant graphs. Electron. Notes Discrete Math. 30, 189-194 (2008)

7. Cerioli, M.R., Korenchendler, A.L.: Clique-coloring circular-arc graphs. Electron. Notes Discrete Math. 35, 287-292 (2009)

8. Charbit, P., Penev, I., Thomassé, S., Trotignon, N.: Perfect graphs of arbitrarily large clique-chromatic number. J. Combin. Theory Ser. B 116, 456-464 (2016) 
9. Chudnovsky, M., Lo, I.: Decomposing and clique-coloring (diamond, odd-hole)-free graphs. J. Graph Theory 86(1), 5-41 (2017)

10. Cochefert, M., Kratsch, D.: Exact algorithms to clique-colour graphs. In: Geffert, V., Preneel, B., Rovan, B., Stuller, J., Tjoa, A.M. (eds.) Proceedings of the 40th International Conference on Current Trends in Theory and Practice of Computer Science (SOFSEM 2014), LNCS, vol. 8327, pp. 187-198. Springer, New York (2014)

11. Courcelle, B., Engelfriet, J., Rozenberg, G.: Handle-rewriting hypergraph grammars. J. Comput. Syst. Sci. 46(2), 218-270 (1993)

12. Courcelle, B., Olariu, S.: Upper bounds to the clique width of graphs. Discrete Appl. Math. 101(1-3), 77-114 (2000)

13. Cygan, M., Dell, H., Lokshtanov, D., Marx, D., Nederlof, J., Okamoto, Y., Paturi, R., Saurabh, S., Wahlström, M.: On problems as hard as CNF-SAT. ACM Transactions on Algorithms 12(3), 41:141:24 (2016)

14. Cygan, M., Fomin, F.V., Kowalik, Ł, Lokshtanov, D., Marx, D., Pilipczuk, M., Pilipczuk, M., Saurabh, S.: Parameterized Algorithms. Springer, New York (2015)

15. Défossez, D.: Clique-coloring some classes of odd-hole-free graphs. J. Graph Theory 53(3), 233-249 (2006)

16. Downey, R.G., Fellows, M.R.: Fundamentals of Parameterized Complexity. Springer, New York (2013)

17. Duffus, D., Sands, B., Sauer, N., Woodrow, R.E.: Two-colouring all two-element maximal antichains. J. Combin. Theory Ser. A 57(1), 109-116 (1991)

18. Fomin, F.V., Golovach, P.A., Lokshtanov, D., Saurabh, S.: Intractability of clique-width parameterizations. SIAM J. Comput. 39(5), 1941-1956 (2010)

19. Fomin, F.V., Golovach, P.A., Lokshtanov, D., Saurabh, S., Zehavi, M.: Clique-width III: Hamiltonian cycle and the odd case of graph coloring. ACM Trans. Algorithms 15(1), 9:1-9:27 (2019)

20. Impagliazzo, R., Paturi, R.: On the complexity of $k$-SAT. J. Comput. Syst. Sci. 62(2), 367-375 (2001)

21. Impagliazzo, R., Paturi, R., Zane, F.: Which problems have strongly exponential complexity? J. Comput. Syst. Sci. 63(4), 512-530 (2001)

22. Jaffke, L., Jansen, B.M.P.: Fine-grained parameterized complexity analysis of graph coloring problems. In: Fotakis, D., Pagourtzis, A., Paschos, V.T. (eds.) Proceedings of the 10th International Conference on Algorithms and Complexity (CIAC 2017), LNCS, vol. 10236, pp. 345-356. Springer (2017)

23. Jaffke, L., Lima, P.T., Lokshtanov, D.: b-Coloring parameterized by clique-width. In: Bläser, M., Monmege, B. (eds.) Proceedings of the 38th International Symposium on Theoretical Aspects of Computer Science (STACS 2021), LIPIcs, vol. 187, pp. 43:1-43:15. Schloss Dagstuhl - LeibnizZentrum für Informatik, Dagstuhl, Germany (2021)

24. Jaffke, L., Lima, P.T., Philip, G.: Structural parameterizations of clique coloring. In: Esparza, J., Král', D. (eds.) Proceedings of the 45th International Symposium on Mathematical Foundations of Computer Science (MFCS 2020), LIPIcs, vol. 170, pp. 49:1-49:15. Schloss Dagstuhl (2020)

25. Klein, S., Morgana, A.: On clique-colouring of graphs with few $P_{4}$ 's. J. Braz. Comput. Soc. 18(2), 113-119 (2012)

26. Kloks, T.: Treewidth: Computations and approximations, LNCS, vol. 842. Springer, New York (1994)

27. Kratochvíl, J., Tuza, Z.: On the complexity of bicoloring clique hypergraphs of graphs. J. Algorithms 45(1), 40-54 (2002)

28. Lokshtanov, D., Marx, D., Saurabh, S.: Known algorithms on graphs of bounded treewidth are probably optimal. ACM Trans. Algorithms 14(2), 13:1-13:30 (2018)

29. Lovász, L.: Combinatorial Problems and Exercises. North-Holland Publishing Co., London (1993)

30. Marx, D.: Complexity of clique coloring and related problems. Theoret. Comput. Sci. 412(29), 3487$3500(2011)$

31. Mohar, B., Škrekovski, R.: The Grötzsch theorem for the hypergraph of maximal cliques. Electron. J. Combin. 6(1), 128 (1999)

32. Penev, I.: Perfect graphs with no balanced skew-partition are 2-clique-colorable. J. Graph Theory 81(3), 213-235 (2016)

33. Rao, M.: Décompositions de graphes et algorithmes efficaces. Ph.D. thesis, University of Metz (2006)

34. Rao, M.: Clique-width of graphs defined by one-vertex extensions. Discrete Math. 308(24), 6157-6165 (2008)

35. Shan, E., Liang, Z., Kang, L.: Clique-transversal sets and clique-coloring in planar graphs. Eur. J. Comb. 36, 367-376 (2014) 
36. Vassilevska, W.V.: Hardness of easy problems: basing hardness on popular conjectures such as the strong exponential time hypothesis (invited talk). In: Husfeldt, T., Kanj, I.A. (eds.) Proceedings of the 10th International Symposium on Parameterized and Exact Computation (IPEC 2015), LIPIcs, vol. 43, pp. 17-29. Schloss Dagstuhl, New York (2015)

Publisher's Note Springer Nature remains neutral with regard to jurisdictional claims in published maps and institutional affiliations. 